\title{
A $\lambda=3 \mathrm{~mm}$ molecular line survey of NGC 1068
}

\section{Chemical signatures of an AGN environment ${ }^{\star, \star \star}$}

\author{
R. Aladro ${ }^{1,2}$, S. Viti ${ }^{2}$, E. Bayet ${ }^{3}$, D. Riquelme ${ }^{4}$, S. Martín ${ }^{1}$, R. Mauersberger ${ }^{5}$, J. Martín-Pintado $^{6}$, \\ M. A. Requena-Torres ${ }^{4}$, C. $\mathrm{Kramer}^{7}$, and A. Weiß ${ }^{4}$
}

\author{
1 European Southern Observatory, Avda. Alonso de Córdova 3107, Vitacura, Santiago, Chile \\ e-mail: raladro@eso.org \\ 2 University College London (UCL), Dept. of Physics \& Astronomy, Gower Street, London WC1E 6BT, UK \\ 3 Sub-Department of Astrophysics, University of Oxford, Denys Wilkinson Building, Keble Road, Oxford OX1 3RH, UK \\ ${ }^{4}$ Max-Planck-Institut für Radioastronomie, Auf dem Hügel 69, 53121 Bonn, Germany \\ 5 Joint ALMA Observatory, Avda. Alonso de Córdova 3107, Vitacura, Santiago, Chile \\ 6 Centro de Astrobiología (CSIC-INTA), Ctra. de Torrejón Ajalvir km 4, 28850 Torrejón de Ardoz, Madrid, Spain \\ 7 Instituto de Radioastronomía Milimétrica, Avda. Divina Pastora, 7, Local 20, 18012 Granada, Spain
}

Received 30 July 2012 / Accepted 12 October 2012

\section{ABSTRACT}

\begin{abstract}
Aims. We study the molecular composition of the interstellar medium (ISM) surrounding an active galactic nucleus (AGN), by making an inventory of molecular species and their abundances, to establish a chemical differentiation between starburst galaxies and AGN. Methods. We used the IRAM-30 m telescope to observe the central 1.5-2 kpc region of NGC 1068, covering the frequencies between 86.2 $\mathrm{GHz}$ and 115.6 GHz. Using Boltzmann diagrams, we calculated the column densities of the detected molecules. We used a chemical model to reproduce the abundances found in the AGN, to determine the origin of each detected species, and to test the influence of UV fields, cosmic rays, and shocks on the ISM.

Results. We identified 24 different molecular species and isotopologues, among which $\mathrm{HC}_{3} \mathrm{~N}, \mathrm{SO}, \mathrm{N}_{2} \mathrm{H}^{+}, \mathrm{CH}_{3} \mathrm{CN}, \mathrm{NS},{ }^{13} \mathrm{CN}$, and $\mathrm{HN}^{13} \mathrm{C}$ are detected for the first time in NGC 1068. A comparison of the abundances in the nuclear regions of NGC 1068, M 82, and NGC 253 allowed us to establish a chemical differentiation between starburst galaxies and AGN. Two abundant species in starburst galaxies, $\mathrm{H}_{2} \mathrm{CO}$ and $\mathrm{CH}_{3} \mathrm{CCH}$, are not detected in NGC 1068, probably because they are destroyed by UV fields or shocks. On the other hand, species such as $\mathrm{CN}, \mathrm{SiO}, \mathrm{HCO}^{+}$, and $\mathrm{HCN}$, are enhanced by cosmic ray radiation fields. We obtained the upper limits to the isotopic ratios ${ }^{12} \mathrm{C} /{ }^{13} \mathrm{C}=49,{ }^{16} \mathrm{O} /{ }^{18} \mathrm{O}=177$, and ${ }^{32} \mathrm{~S} /{ }^{34} \mathrm{~S}=5$. These ratios are much lower in this AGN than in starburst galaxies. Our chemical models suggest that the chemistry in the nucleus of NGC 1068 is strongly influenced by cosmic rays, although high values of both cosmic rays and far ultraviolet (FUV) radiation fields also explain the observations well. C-shocks can explain the abundances of $\mathrm{C}_{2} \mathrm{H}$ and $\mathrm{H}_{2} \mathrm{CO}$, but do not strongly affect the abundances of the other detected species.

Conclusions. The gas in the nucleus of NGC 1068 has a different chemical composition than starburst galaxies. The distinct physical processes dominating galaxy nuclei (e.g. C-shocks, UV fields, X-rays, cosmic rays) leave clear imprints in the chemistry of the gas, which allow the nucleus activity to be characterised by its molecular abundances.
\end{abstract}

Key words. ISM: molecules - galaxies: ISM - galaxies: individual: NGC 1068 - galaxies: nuclei - galaxies: active

\section{Introduction}

NGC 1068 is one of the closest $\left(D=14.4 \mathrm{Mpc}, 1^{\prime \prime}=72 \mathrm{pc}\right.$, Bland-Hawthorn et al. 1997) Seyfert 2 galaxies. It has an infrared (IR) luminosity of $3 \times 10^{11} L_{\odot}$ (Telesco \& Harper 1980). The IR observations reveal warm dust with temperatures of $320 \mathrm{~K}$ surrounding a smaller hot structure (Jaffe et al. 2004) assumed to be a central black hole of mass $1.7 \times 10^{7} M_{\odot}$ (Schinnerer et al. 2000), and CO observations show molecular spiral arms forming a starburst ring $10^{\prime \prime}$ from the nucleus. Inside the ring, NGC 1068 has a circumnuclear disk (CND) with a thickness of $10 \mathrm{pc}$ (Schinnerer et al. 2000, see Fig. 1).

NGC 1068 is therefore one of the best extragalactic targets for studying the physical and chemical properties of the interstellar medium (ISM) in the vicinity of an active galactic

\footnotetext{
Appendices are available in electronic form at http: //www . aanda.org

$\star \star$ FITS files of the spectra are only available at the CDS via anonymous ftp to cdsarc.u-strasbg. fr $(130.79 .128 .5)$ or via http://cdsarc.u-strasbg.fr/viz-bin/qcat?]/A+A/549/A39
}

nucleus (AGN). The molecular material close to the central engines of AGN is pervaded by $\mathrm{X}$-ray and cosmic ray radiations originating in the nuclear accretion disks (Maloney et al. 1996). Previous single-dish and interferometric observations of NGC 1068 in a number of molecules, such as $\mathrm{CO}, \mathrm{SiO}$, $\mathrm{HOC}^{+}$, and $\mathrm{CN}$, have revealed a chemistry interpreted as the result of a giant X-ray-dominated region (XDR) in its nucleus, where shocks could also be heating the gas (Usero et al. 2004; García-Burillo et al. 2010; Kamenetzky et al. 2011). This strong $\mathrm{X}$-ray radiation has also been claimed to be responsible for the enhanced $\mathrm{HCN}$-to- $\mathrm{HCO}^{+}$line ratio, which differs between AGN and starburst environments (Kohno et al. 2001; Krips et al. 2008). However, a comprehensive study of the molecular content of the ISM close to AGNs has not been carried out so far. As a consequence, the influence of $\mathrm{X}$-rays and cosmic rays on the molecular gas is only partially understood.

Unbiased molecular line surveys done toward the nuclei of the starburst galaxies NGC 253 (Martín et al. 2006) and M 82 (Aladro et al. 2011b) have allowed determination of the impact of large-scale shocks and UV fields on the ISM, as well 


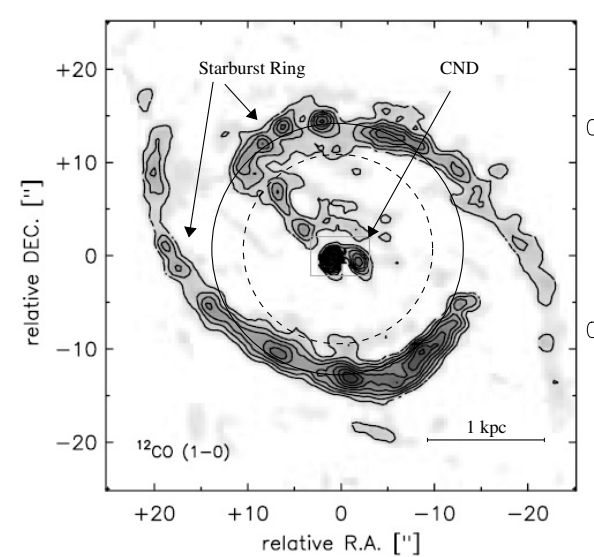

(a)

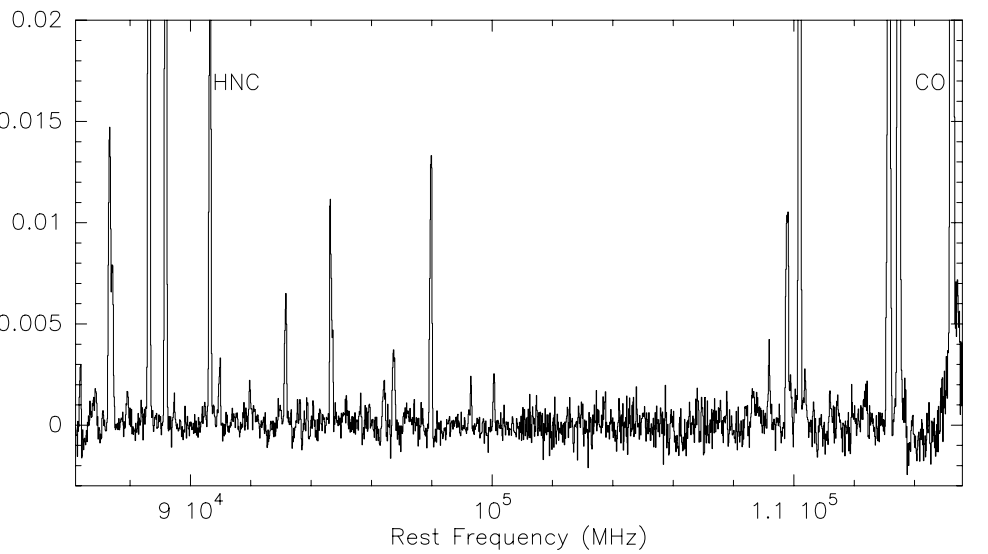

(b)

Fig. 1. a) ${ }^{12} \mathrm{CO}(1-0)$ map of the NGC 1068 nucleus (taken from Schinnerer et al. 2000). The beam sizes at the lowest and highest frequencies of the survey are plotted with a solid and a dotted circle respectively. The starburst ring and CND are also indicated. b) Our molecular line survey in the $3 \mathrm{~mm}$ wavelengths. Temperatures are in $T_{\mathrm{MB}}(\mathrm{K})$.

as analysis of how its composition changes as the starburst phenomena evolve with time. It is clear from these studies that a detailed molecular inventory description of a prototypical AGN is also needed in order to understand the properties of extragalactic XDRs, and also to chemically differentiate between nuclear powering sources (starbursts versus AGN).

In this work we present a comprehensive chemical study of the gas in the NGC 1068 nucleus. We characterise its physical and chemical properties and highlight some important differences with the massive star-forming regions in starburst galaxies. In Sects. 2 and 3 we present details of the observations, data reduction, and analysis. Section 4 shows the results obtained from the survey and a discussion of the NGC 1068 chemistry. In Sect. 5 we present a chemical model of NGC 1068 used to investigate how UV radiation, cosmic rays, and C-type shocks may affect its gas. We also discuss the origin of each detected species and present the comparison of the model results with the observational data. In Sect. 6 we compare the properties of the circumnuclear gas in NGC 1068 with those found in starburst galaxies. Finally, we present our conclusions in Sect. 7.

\section{Observations and data reduction}

The observations were carried out with the IRAM-30 m telescope $^{1}$ (Pico Veleta Observatory, Spain) between October 2009 and July 2010. We observed the nucleus of NGC 1068, at a nominal position $\alpha_{2000}=02: 42: 40.9, \delta_{2000}=-00: 00: 46.0$, between the frequencies 86.2 and $115.6 \mathrm{GHz}$. The assumed heliocentric systemic velocity was $1110 \mathrm{~km} \mathrm{~s}^{-1}$. The half-power beam width (HPBW) ranged from $29^{\prime \prime}$ to $21^{\prime \prime}$, corresponding to a spatial scale of $2-1.5 \mathrm{kpc}$. We used the band E0 of the EMIR receiver and the WILMA autocorrelator. This receiver-backend configuration allowed us to cover $8 \mathrm{GHz}$ simultaneously in the vertical and horizontal polarisations, and led to a channel-width spacing of 7-9 $\mathrm{km} \mathrm{s}^{-1}$. The IRAM-30 $\mathrm{m}$ data were first calibrated to the antenna temperature $\left(T_{\mathrm{A}}^{*}\right)$ scale using the chopperwheel method (Penzias \& Burrus 1973). The observations were done by wobbling the secondary mirror with a switching frequency of $0.5 \mathrm{~Hz}$ and a beam throw of $110^{\prime \prime}$ in azimuth. We checked the pointing accuracy every hour towards the nearby

\footnotetext{
1 IRAM is supported by INSU/CNRS (France), MPG (Germany), and IGN (Spain).
}

bright continuum sources $2251+158$ and $0113-118$. The pointing corrections were always better than $4^{\prime \prime}$. The focus was also checked at the beginning of each run and during sunsets.

The observed spectra were converted from $T_{\mathrm{A}}^{*}$ to main beam temperatures $\left(T_{\mathrm{MB}}\right)$ using the relation $T_{\mathrm{MB}}=\left(F_{\text {eff }} / B_{\mathrm{eff}}\right) T_{\mathrm{A}}^{*}$, where $F_{\text {eff }}$ is the forward efficiency of the telescope, whose values were between 0.94 and 0.95 , and $B_{\text {eff }}$ is the main beam efficiency, ranging from 0.77 to 0.81 . Linear baselines were subtracted in all cases. We note that some of the spectra still contain faint ripples that were not completely removed by our switching scheme. This results in some of the weak features observed (e.g. around $94 \mathrm{GHz}, 111.3 \mathrm{GHz}$ and $112.3 \mathrm{GHz}$ ). However, these features are quite below the noise of the spectra. The rms achieved is $\leq 2 \mathrm{mK}$ ( $\leq 10 \mathrm{mJy} /$ beam) across the whole survey. The survey coverage is shown in Fig. 1b, while more detailed spectra, where the detected species are labelled, are shown in Fig. 2.

The data were also corrected to first order for beam dilution effects as $T_{\mathrm{B}}=\left[\left(\theta_{\mathrm{s}}^{2}+\theta_{\mathrm{b}}^{2}\right) / \theta_{\mathrm{s}}^{2}\right] T_{\mathrm{MB}}$, where $T_{\mathrm{B}}$ is the source averaged brightness temperature, $\theta_{\mathrm{s}}$ is the source size, and $\theta_{\mathrm{b}}$ the beam size. Based on NGC 1068 interferometric observations of ${ }^{12} \mathrm{CO}, \mathrm{HCN}$, and ${ }^{13} \mathrm{CO}$ (e.g. Helfer \& Blitz 1995; Schinnerer et al. 2000), we assumed an average source size of 4 " for all the species detected in this survey. This value is consistent with other studies focused on the CND of NGC 1068 (e.g. Bayet et al. 2009b; Krips et al. 2008, 2011; Kamenetzky et al. 2011).

Gaussian profiles were fitted to all the detected lines. The parameters resulting from those fits are given in Table B.1. The reduction of the spectra and Gaussian profile fitting were done using the CLASS ${ }^{2}$ and MASSA ${ }^{3}$ software packages. In those cases where there were two or more molecules blended, we performed a synthetic fitting (see Martín et al. 2010; Aladro et al. 2011b, for details about this method) in order to calculate the contribution of each line to the total profile. Each case is discussed further in Appendix A.

\section{Analysis of the data}

The line identification was made using the CDMS (Müller et al. 2001, 2005), JPL (Pickett et al. 1998) and LOVAS (Lovas 1992)

\footnotetext{
2 CLASS http://wWw.iram.fr/IRAMFR/GILDAS

3 MASSA

http://damir.iem.csic.es/mediawiki-1.12.0/index.php/ MASSA_User's_Manual
} 

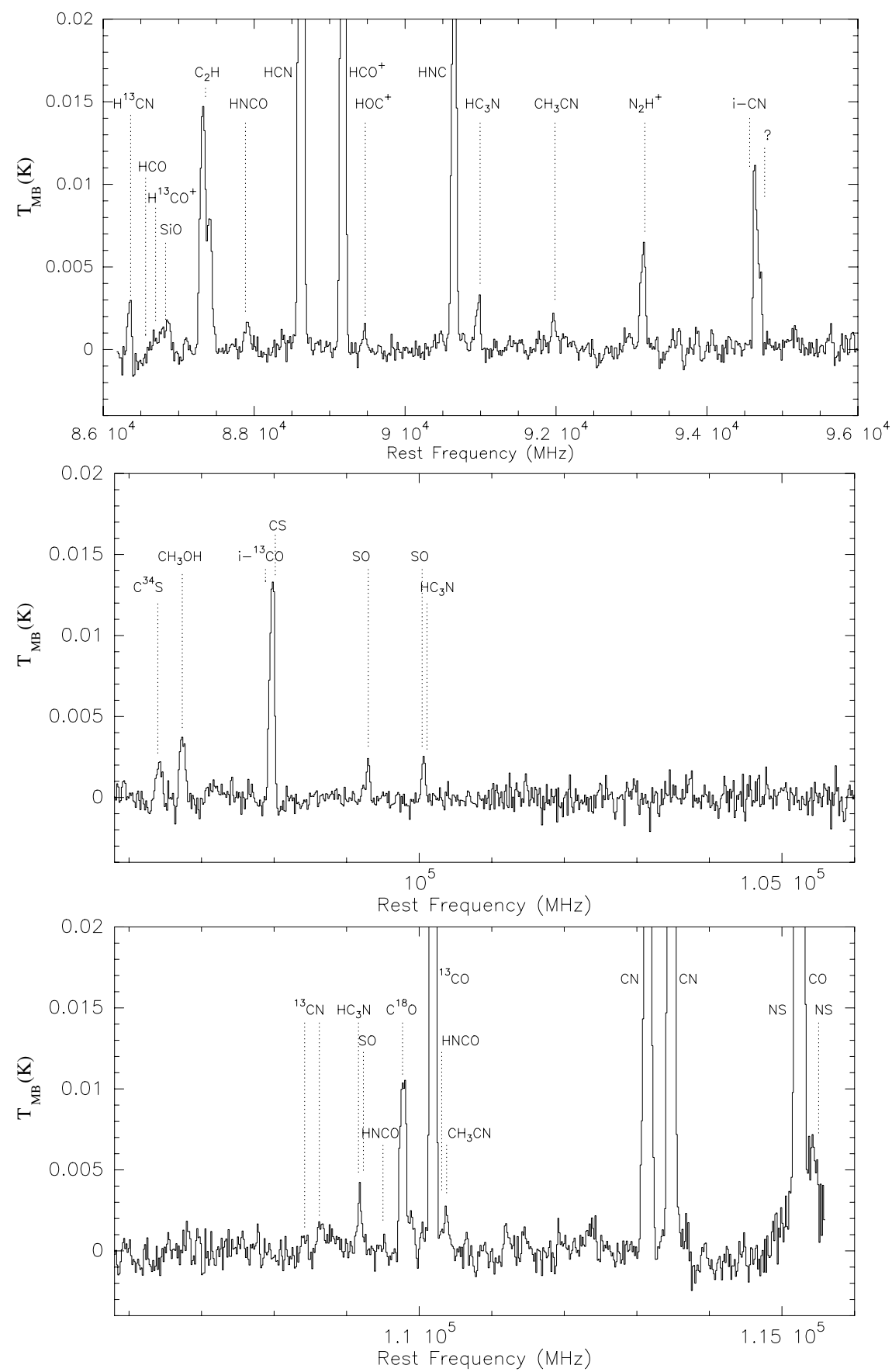

Fig. 2. NGC 1068 frequency survey coverage between 86.2 and $115.6 \mathrm{GHz}$. The spectra have been smoothed to a velocity resolution of $\sim 44 \mathrm{~km} \mathrm{~s}^{-1}$ (corresponding to $\sim 16 \mathrm{MHz}$ ). The assumed systemic velocity is $1110 \mathrm{~km} \mathrm{~s}^{-1}$.

catalogues, and it largely follows the method explained in detail in Aladro et al. (2011b) for a similar line survey done toward the nucleus of M 82. The image band rejection of the EMIR receiver was better than $10 \mathrm{~dB}$ along the band, so only ${ }^{13} \mathrm{CO}(1-0)$ and $\mathrm{CN}(1-0)$ entered from the image band, at $97.9 \mathrm{GHz}$ and $94.6 \mathrm{GHz}$, respectively. The latter is blended with one unidentified feature. No strong line is expected at $\sim 94.6 \mathrm{GHz}$, and the $\mathrm{CN}(1-0)$ line coming from the image band cannot account for the total emission. On the other hand, the ${ }^{13} \mathrm{CO}(1-0)$ line coming from the image band is blended with CS (2-1). Since there are no other CS lines in our survey, and the exact rejection of the image band at this particular frequency is not known, we cannot calculate the intensity of the CS transition. Unfortunately, it has not been observed previously, so we cannot take it from the literature. However, other CS transitions have been observed towards the nucleus of NGC 1068 by Bayet et al. (2009b), so we include them in our study.

\subsection{Contribution of the starburst ring}

As shown in Fig. 1a, the angular resolution of the IRAM-30 m telescope at $3 \mathrm{~mm}$ wavelengths is not high enough to separate the CND of NGC 1068 from the surrounding starburst ring. As a consequence, some of the detected molecules in our survey might include some emission of the starburst ring picked up the telescope beam.

The $J-J^{\prime}=1-0$ transitions of $\mathrm{HCO}^{+}, \mathrm{HNC}, \mathrm{C}^{18} \mathrm{O},{ }^{13} \mathrm{CO}$, and ${ }^{12} \mathrm{CO}$ are deviated from a Gaussian profile. Instead, they show emission in the wings, and the lines are better fitted by three Gaussian components. As an example, we show in Fig. A.2 
the profile of the $\mathrm{CO}(1-0)$ line. The gas dynamics in the centre of NGC 1068 and, in particular, in the CND are quite complex. Interferometric observations have already shown that the profiles of $\mathrm{CO}, \mathrm{HCN}$, and $\mathrm{HCO}^{+}$lines need dual, triple, or even quadrupole Gaussian fits in order to reproduce their profiles better (Krips et al. 2011). This was associated to non-circular motions in the central $\sim 100 \mathrm{pc}$, where part of the gas might be blown outward due to shocks (Krips et al. 2011), while the rest of the gas is accreted toward the AGN along the jet (e.g. Müller Sánchez et al. 2009).

It is not possible, with single-dish observations, to disentangle whether the deviations from the Gaussian profiles are due only to the complex gas dynamics or if the starburst ring emission is also contributing to the line shapes. Furthermore, for other species no cited above there are other factors that make it even more difficult to evaluate the possible starburst ring contribution. For example, some molecules such as $\mathrm{C}_{2} \mathrm{H}$ and $\mathrm{HCN}$, have a hyperfine structure that intrinsically creates deviations from Gaussian profiles. Others, such as $\mathrm{H}^{13} \mathrm{CO}^{+}, \mathrm{HC}_{3} \mathrm{~N}$, or $\mathrm{SiO}$, are blended in our survey or are very weak in NGC 1068, so a multiple Gaussian fit is not feasible. Therefore, since we cannot correct for the starburst emission, the data we show in the following sections include all the radiation picked up by the telescope beam (i.e. CND plus starburst ring).

\subsection{LTE analysis}

Assuming local thermodynamic equilibrium (LTE) and optically thin emission for all the detected species, we made Boltzmann diagrams to estimate the rotational temperatures $\left(T_{\text {rot }}\right)$ and column densities of the molecules $\left(N_{\text {mol }}\right)$. A detailed explanation of the method can be found in Goldsmith \& Langer (1999). The rotation diagrams are shown in Fig. A.3, while the resulting parameters are listed in Table 1. To determine the rotational temperatures from the Boltzmann plots, at least two transitions of a given molecule are needed. In those cases where only one transition of a molecule lies in our $3 \mathrm{~mm}$ survey $\left(\mathrm{CO},{ }^{13} \mathrm{CO}\right.$, $\mathrm{C}^{18} \mathrm{O}, \mathrm{CS}, \mathrm{CN}, \mathrm{HCO}^{+}, \mathrm{HNC}$, and $\mathrm{C}^{34} \mathrm{~S}$ ), we complemented our observations with other lines, if available, in the literature (see Table 1 for references). For ${ }^{13} \mathrm{CN}, \mathrm{H}^{13} \mathrm{CN}, \mathrm{H}^{13} \mathrm{CO}^{+}$, and $\mathrm{HN}^{13} \mathrm{C}$ there are no previous data published, so we simply assumed that these species arise from gas with the same temperature as the main ${ }^{12} \mathrm{C}$ isotopologues. We also considered the same temperature for $\mathrm{HOC}^{+}$and $\mathrm{HCO}^{+}$. For the rest of the cases $\left(\mathrm{C}_{2} \mathrm{H}, \mathrm{HCO}\right.$, $\mathrm{NS}, \mathrm{N}_{2} \mathrm{H}^{+}$) with more than one transition neither in our survey nor in the literature, we fixed a $T_{\text {rot }}=10 \pm 5 \mathrm{~K}$, which is an average value of the rotational temperatures determined in this survey.

As mentioned before, the rotation diagrams were done assuming optically thin emission of the gas. However, some molecules are certainly affected by optical thickness (e.g. CO, HCN, Jackson et al. 1993; Papadopoulos \& Seaquist 1999). Therefore, in these cases we are underestimating the actual column densities. The optical depths of the most intense molecules in the survey are discussed further in Sect. 4.3.

\section{Results}

\subsection{Detected and undetected molecules}

We detected a total of 35 lines, or groups of lines, in our survey, which correspond to 17 different molecular species and seven isotopologues. Some of them were detected for the first time in this galaxy, namely $\mathrm{HC}_{3} \mathrm{~N}, \mathrm{SO}, \mathrm{N}_{2} \mathrm{H}^{+}, \mathrm{CH}_{3} \mathrm{CN}, \mathrm{NS},{ }^{13} \mathrm{CN}$,
Table 1. Column densities and rotational temperatures of the observed molecules.

\begin{tabular}{|c|c|c|c|}
\hline Molecule & $\begin{array}{c}T_{\text {rot }^{\dagger}} \\
\mathrm{K}\end{array}$ & $\begin{array}{l}N_{\text {mol }} \\
\mathrm{cm}^{-2}\end{array}$ & Ref. \\
\hline \multirow[t]{2}{*}{${ }^{12} \mathrm{CO}$} & $5.0 \pm 0.3$ & $(3.8 \pm 0.3) \times 10^{18}$ & $\mathrm{a}$ \\
\hline & $38.4 \pm 6.5$ & $(1.2 \pm 0.3) \times 10^{18}$ & $\mathrm{a}$ \\
\hline \multirow[t]{2}{*}{${ }^{13} \mathrm{CO}$} & $4.3 \pm 0.2$ & $(3.2 \pm 0.3) \times 10^{17}$ & $\mathrm{a}$ \\
\hline & $24.1 \pm 6.3$ & $(7.8 \pm 2.8) \times 10^{16}$ & $\mathrm{a}$ \\
\hline $\mathrm{C}^{18} \mathrm{O}$ & $3.3 \pm 0.1$ & $(1.0 \pm 0.1) \times 10^{17}$ & $\mathrm{~b}$ \\
\hline $\mathrm{C}_{2} \mathrm{H}$ & $10.0 \pm 5.0$ & $(1.0 \pm 0.5) \times 10^{16}$ & \\
\hline \multirow[t]{2}{*}{$\mathrm{CN}$} & $8.8 \pm 0.1$ & $(5.0 \pm 0.1) \times 10^{15}$ & d \\
\hline & $51.4 \pm 36.0$ & $(5.8 \pm 4.4) \times 10^{15}$ & d \\
\hline $\mathrm{CH}_{3} \mathrm{OH}$ & $5.6 \pm 0.1$ & $(2.3 \pm 0.1) \times 10^{15}$ & \\
\hline $\mathrm{HCN}$ & $5.0 \pm 0.3$ & $(1.4 \pm 0.2) \times 10^{15}$ & $\mathrm{e}$ \\
\hline \multirow[t]{2}{*}{$\mathrm{CS}$} & $7.1 \pm 0.4$ & $(7.6 \pm 2.2) \times 10^{14}$ & $\mathrm{c}$ \\
\hline & $33.0 \pm 13.1$ & $(7.0 \pm 4.2) \times 10^{13}$ & $\mathrm{c}$ \\
\hline $\mathrm{HC}_{3} \mathrm{~N}$ & $7.3 \pm 1.4$ & $(6.0 \pm 4.0) \times 10^{14}$ & \\
\hline $\mathrm{HCO}^{+}$ & $4.9 \pm 0.1$ & $(5.3 \pm 0.2) \times 10^{14}$ & $\mathrm{e}$ \\
\hline HNC & $7.6 \pm 0.2$ & $(4.9 \pm 0.5) \times 10^{14}$ & d \\
\hline $\mathrm{HCO}$ & $10.0 \pm 5.0$ & $(2.1 \pm 1.3) \times 10^{14}$ & \\
\hline SO & $22.8 \pm 18.6$ & $(3.2 \pm 3.3) \times 10^{14}$ & \\
\hline NS & $10.0 \pm 5.0$ & $(2.8 \pm 1.4) \times 10^{14}$ & \\
\hline $\mathrm{HNCO}$ & $29.7 \pm 0.1$ & $(2.8 \pm 0.1) \times 10^{14}$ & \\
\hline${ }^{13} \mathrm{CN}$ & $8.8 \pm 0.1$ & $(2.2 \pm 0.1) \times 10^{14}$ & \\
\hline $\mathbf{N}_{2} \mathbf{H}^{+}$ & $10.0 \pm 5.0$ & $(1.1 \pm 0.5) \times 10^{14}$ & \\
\hline $\mathrm{C}^{34} \mathrm{~S}$ & $3.6 \pm 0.5$ & $(1.6 \pm 0.6) \times 10^{14}$ & $\mathrm{~b}$ \\
\hline $\mathrm{H}^{13} \mathrm{CN}$ & $5.0 \pm 0.3$ & $(8.7 \pm 0.5) \times 10^{13}$ & \\
\hline $\mathrm{SiO}$ & $3.8 \pm 0.2$ & $(6.4 \pm 0.8) \times 10^{13}$ & $\mathrm{x}$ \\
\hline $\mathrm{CH}_{3} \mathrm{CN}$ & $10.2 \pm 0.1$ & $(3.9 \pm 0.1) \times 10^{13}$ & \\
\hline $\mathrm{H}^{13} \mathrm{CO}^{+}$ & $4.9 \pm 0.1$ & $(3.1 \pm 0.1) \times 10^{13}$ & \\
\hline $\mathrm{HN}^{13} \mathrm{C}$ & $7.6 \pm 0.2$ & $(2.1 \pm 3.2) \times 10^{13}$ & \\
\hline $\mathrm{HOC}+$ & $4.9 \pm 0.1$ & $(1.1 \pm 0.6) \times 10^{13}$ & \\
\hline $\mathrm{c}-\mathrm{C}_{3} \mathrm{H}_{2}$ & 7.6 & $(1.7 \pm \ldots) \times 10^{13}$ & $\mathrm{f}$ \\
\hline $\mathrm{NH}_{3}$ & & $(1.1 \pm 0.2) \times 10^{14}$ & $\mathrm{~g}$ \\
\hline
\end{tabular}

Notes. Molecules in boldface are new detections in NGC 1068. The values given in this table are already corrected by beam dilution effects. (†) For those molecules with only one rotational transition available, we assumed $T_{\text {rot }}=10 \pm 5 \mathrm{~K}$. See Sect. 2 for details.

References. Last column refers to detections taken from the literature: (a) Israel (2009); (b) Martín et al. (2009); (c) Bayet et al. (2009b); (d) Pérez-Beaupuits et al. (2009); (e) Krips et al. (2008); (x) Usero et al. (2004). c- $\mathrm{C}_{3} \mathrm{H}_{2}$ and $\mathrm{NH}_{3}$ were not detected in this survey, but were compiled from the literature; (f) Nakajima et al. (2011); and (g) Ao et al. (2011).

and $\mathrm{HN}^{13} \mathrm{C}$. As detailed in Sect. 2, we complemented our observations with other detections taken from the literature (see Table 1 for references). From the Boltzmann diagram results, ${ }^{12} \mathrm{CO},{ }^{13} \mathrm{CO}, \mathrm{CN}$, and $\mathrm{CS}$ show two temperature components, the warmer one coming from the higher $J-J^{\prime}$ transitions compiled from the literature (marked in Fig. A.3 with an asterisk).

We calculated column density ratios that are said to be representative of AGNs, as opposed to starburst environments. The NGC 1068 chemistry can be summarised as follows: high $N_{\mathrm{HCN}} / N_{\mathrm{CO}}\left(\sim 3 \times 10^{-4}\right)$, high $N_{\mathrm{CN}} / N_{\mathrm{HCN}}(\sim 3-4)$, low $N_{\mathrm{HCO}^{+}} / N_{\mathrm{HCN}}(\sim 0.4)$, and low $N_{\mathrm{HCO}^{+}} / N_{\mathrm{HOC}^{+}}(\sim 48)$. These results are consistent with many previous studies, such as Usero et al. (2004), Pérez-Beaupuits et al. (2009), and Krips et al. (2008, 2011). A comparison of these and other molecular ratios with those typically found in starburst galaxies is presented in Sect. 6.

We also list in Table 2 molecules that were not detected in our survey, but that were successfully observed in other extragalactic sources. We show the $3 \sigma$ upper limits to their column 
Table 2. Upper limits to the column densities of some undetected species.

\begin{tabular}{lccc}
\hline \hline Molecule & $\begin{array}{c}N_{\text {mol }} \\
\mathrm{cm}^{-2}\end{array}$ & Molecule & $\begin{array}{c}N_{\text {mol }} \\
\mathrm{cm}^{-2}\end{array}$ \\
\hline $\mathrm{H}_{2} \mathrm{CO}$ & $\leq 1.1 \times 10^{16}$ & $\mathrm{H}_{2} \mathrm{CS}$ & $\leq 2.6 \times 10^{14}$ \\
$\mathrm{OCS}$ & $\leq 5.4 \times 10^{14}$ & $\mathrm{C}_{2} \mathrm{~S}$ & $\leq 8.9 \times 10^{13}$ \\
$\mathrm{CH}_{2} \mathrm{NH}$ & $\leq 5.4 \times 10^{14}$ & $\mathrm{HOCO}^{+}$ & $\leq 7.0 \times 10^{13}$ \\
$\mathrm{CH}_{3} \mathrm{CCH}$ & $\leq 4.5 \times 10^{14}$ & ${ }^{13} \mathrm{CS}$ & $\leq 3.6 \times 10^{13}$ \\
$\mathrm{SO}_{2}$ & $\leq 3.6 \times 10^{14}$ & $\mathrm{NH}_{2} \mathrm{CN}$ & $\leq 2.8 \times 10^{13}$ \\
$\mathrm{c} \mathrm{C}_{3} \mathrm{H}$ & $\leq 2.9 \times 10^{14}$ & & \\
\hline
\end{tabular}

Notes. See Sect. 4 for details about the calculations.

densities, which were derived from the rms noise level of the corresponding spectra. Some of the non-detected species in NGC 1068 , such as methyl acetylene $\left(\mathrm{CH}_{3} \mathrm{CCH}\right)$ or formaldehyde $\left(\mathrm{H}_{2} \mathrm{CO}\right)$, are quite abundant in starburst galaxies. These two molecules are presumably too faint to be detected in NGC 1068, because other studies, also covering wide ranges of frequencies, did not detect them either (e.g. Kamenetzky et al. 2011). We discuss in Sect. 5.3 possible reasons for their weakness.

\subsection{Rotational temperatures}

The rotational temperatures obtained from the Boltzmann diagrams (for those species with more than one detected transition) range between $\sim 3$ and $\sim 51 \mathrm{~K}$ (see Table 1 ). These values are far below the kinetic gas temperature $\left(T_{\text {kin }}\right)$ traced by ammonia $\left(\mathrm{NH}_{3}\right.$ ), of $80 \pm 20 \mathrm{~K}$, for the bulk of the molecular gas (Ao et al. 2011). However, we note that $T_{\text {rot }}$ are always lower limits to $T_{\text {kin }}$, and that the conversion from rotational to kinetic temperatures is not simple. Also, the data we use seem to be subthermally excited.

Our results agree well with previous studies where it was found that low- $J \mathrm{CO}, \mathrm{HCN}$, and $\mathrm{HCO}^{+}$lines trace cold gas $\left(T_{\mathrm{kin}}^{\mathrm{HCO}^{+}} \leq 20 \mathrm{~K}\right.$, Pérez-Beaupuits et al. $2009 ; T_{\mathrm{kin}}^{\mathrm{CO}} \approx 30 \mathrm{~K}$, Israel $2009 ; T_{\mathrm{kin}}^{\mathrm{CO}, \mathrm{HCN}} \approx 50 \mathrm{~K}$, Sternberg et al. 1994).

\subsection{Isotopic line ratios}

Isotopic ratios are commonly studied through the line ratios between different molecular species and their isotopic substitutions. However, molecular line ratios are often affected by opacity effects, selective photodissociation, or/and isotopic fractionation, thus giving only lower limits to the actual values of isotopic ratios. Fractionation processes could affect the carbon isotopic molecular ratios (but not the oxygen and sulphur ones, Wannier 1980; Woods \& Willacy 2009), and might be more effective in regions with low kinetic temperatures (such as cold dark clouds, Sakai et al. 2010), and may be not negligible for NGC 1068 given the low temperatures traced by some molecules (see Sect. 4.2). On the other hand, in the presence of strong UV radiation, isotope-selective photoionisation can also take place in the external regions of molecular clouds, where shelf-shielding and mutual shielding between different carbon isotopologues can occur (Glassgold et al. 1985). This seems to have a negligible impact on the ${ }^{12} \mathrm{CO} /{ }^{13} \mathrm{CO}$ ratio, although it may modify the $\mathrm{C}^{16} \mathrm{O} / \mathrm{C}^{18} \mathrm{O}$ result $(\mathrm{Chu} \&$ Watson 1983 ; Wilson \& Rood 1994). UV fields may be strong in the nucleus of NGC 1068 (see our modelling results in Sect. 5) so, apart from our ${ }^{12} \mathrm{CO} /{ }^{13} \mathrm{CO}$, we cannot rule out that selective photoionisation is affecting our lower limits to the carbon and oxygen
Table 3. Molecular line ratios in NGC 1068 and comparison with the isotopic ratios in other galaxies.

\begin{tabular}{|c|c|c|}
\hline $\begin{array}{l}\text { NGC } 1068 \\
J-J^{\prime}=1-0\end{array}$ & GC & M 82 \\
\hline $\begin{array}{l}{ }^{12} \mathrm{CO} /{ }^{13} \mathrm{CO}=12.6 \\
\mathrm{H}^{12} \mathrm{CO}^{+} / \mathrm{H}^{13} \mathrm{CO}^{+}=17.1 \\
\mathrm{HN}^{12} \mathrm{C} / \mathrm{HN}^{13} \mathrm{C}=23.3 \\
\mathrm{H}^{12} \mathrm{CN} / \mathrm{H}^{13} \mathrm{CN}=16.1 \\
{ }^{12} \mathrm{CN} /{ }^{13} \mathrm{CN}=49.1\end{array}$ & ${ }^{12} \mathrm{C} /{ }^{13} \mathrm{C} \sim 20-25^{g}$ & ${ }^{12} \mathrm{C} /{ }^{13} \mathrm{C}>138^{e}$ \\
\hline $\begin{array}{l}\mathrm{C}^{16} \mathrm{O} / \mathrm{C}^{18} \mathrm{O}=176.8^{a} \\
\mathrm{C}^{32} \mathrm{~S} / \mathrm{C}^{34} \mathrm{~S}=5.2^{b}\end{array}$ & $\begin{aligned}{ }^{16} \mathrm{O} /{ }^{18} \mathrm{O} & =250^{c} \\
{ }^{32} \mathrm{~S} /{ }^{\beta 4} \mathrm{~S} & \sim 22^{d}\end{aligned}$ & $\begin{aligned} &{ }^{16} \mathrm{O} /{ }^{18} \mathrm{O}>350^{e} \\
&{ }^{32} \mathrm{~S} /{ }^{34} \mathrm{~S}=20^{f}\end{aligned}$ \\
\hline
\end{tabular}

Notes. ${ }^{(a)}$ See details in Sect. 4.3 about how this value was calculated; (b) using data from the literature (see Table 1 for references); ${ }^{(c)}$ Wilson \& Rood (1994); ${ }^{(d)}$ Frerking et al. (1980); ${ }^{(e)}$ Martín et al. (2010), using $\mathrm{C}_{2} \mathrm{H}$ and its two C-isotopologues; ${ }^{(f)}$ Martín et al. (2009) and Aladro et al. (2011a); ${ }^{(g)}$ Wannier (1980).

isotopic ratios. Finally, opacity effects clearly influence our derived ${ }^{12} \mathrm{C} /{ }^{13} \mathrm{C}$ ratios, since they certainly affect the main isotopologues of $\mathrm{CO}, \mathrm{HCO}^{+}$, and $\mathrm{HCN}$ in NGC 1068. In contrast, ${ }^{13} \mathrm{C}$-bearing isotopic species are expected to be optically thin, or only moderately optically thick.

We used the detected transitions of $\mathrm{CO}, \mathrm{HCN}, \mathrm{HNC}, \mathrm{HCO}^{+}$, $\mathrm{CN}$, and their ${ }^{13} \mathrm{C}$ isotopologues to calculate a lower limit to the ${ }^{12} \mathrm{C} /{ }^{13} \mathrm{C}$ ratio in the nucleus of $\mathrm{NGC} 1068$. On the other hand, using the observed lines of $\mathrm{C}^{16} \mathrm{O}, \mathrm{C}^{18} \mathrm{O}, \mathrm{C}^{32} \mathrm{~S}$, and $\mathrm{C}^{34} \mathrm{~S}$, we derived limits to the ${ }^{16} \mathrm{O} /{ }^{18} \mathrm{O}$ and ${ }^{32} \mathrm{~S} /{ }^{34} \mathrm{~S}$ isotopic ratios. Our results are summarised in Table 3. They were calculated by dividing the column densities listed in Table 1.

Our molecular line ratio ${ }^{12} \mathrm{CO}(1-0) /{ }^{13} \mathrm{CO}(1-0)=12.6$ is in accordance with previous results, where moderately optically thickness of $\tau \sim 1-2$ for CO (1-0) was found (Young \& Sanders 1986; Papadopoulos et al. 1996). The $\mathrm{HCO}^{+}(1-0) / \mathrm{H}^{13} \mathrm{CO}^{+}(1-0), \quad \mathrm{HCN}(1-0) / \mathrm{H}^{13} \mathrm{CN}(1-0)$, and $\mathrm{HNC}(1-0) / \mathrm{HN}^{13} \mathrm{C}(1-0)$ ratios give similar values, of $17.1,16.1$ and 23.3, respectively. Among all, $\mathrm{CN}$ is the species giving the highest lower limit to the ${ }^{12} \mathrm{C} /{ }^{13} \mathrm{C}$ ratio, of 49.1 .

Using the $\mathrm{C}^{16} \mathrm{O}(1-0)$ and $\mathrm{C}^{18} \mathrm{O}(1-0)$ column densities, we obtained ${ }^{16} \mathrm{O} /{ }^{18} \mathrm{O}=50.0$. This low value points to the opacity of both $\mathrm{C}^{16} \mathrm{O}(1-0)$ and $\mathrm{C}^{18} \mathrm{O}(1-0)$ lines (where the latter has $\tau \geq 1$, Papadopoulos \& Seaquist 1999). However, if we calculate

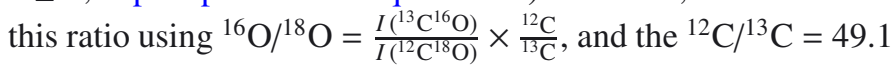
obtained from $\mathrm{CN}$, we obtain a higher lower limit to the ${ }^{16} \mathrm{O} /{ }^{18} \mathrm{O}$ ratio of 176.8 .

Unfortunately, our CS (2-1) line is contaminated by the emission of ${ }^{13} \mathrm{CO}(1-0)$ coming from the image band (see Sect. 3). Therefore, we used the CS (3-2) and $C^{34} S(3-2)$ detections by Martín et al. (2009) and Bayet et al. (2009b) to calculate a limit to ${ }^{32} \mathrm{~S} /{ }^{34} \mathrm{~S}$, which gives a result of 5.2.

\section{Modelling the data}

To model the molecular line emissions presented in Sect. 4, we used the time- and depth-dependent chemical model UCL_CHEM (Viti \& Williams 1999; Viti et al. 2004). The molecular emission from the AGN of NGC 1068 arises from an ensemble of gas components ranging in density and temperature by several orders of magnitudes and representing various stages of the ISM, from diffuse to dense gas. Quantitatively modelling the observed line emission would thus require some knowledge of the geometry, as well as the dynamics, of the different gas components. Instead chemical and photon-dominated 
Table 4. Main parameters of the UCL_CHEM models.

\begin{tabular}{lccccc}
\hline \hline Parameter & Model $a$ & Model $b$ & Model $c$ & Model $d$ & Model $e$ \\
\hline Temperature (phase II) & $200 / 100 \mathrm{~K}$ & $300 / 100 \mathrm{~K}$ & $250 / 300 \mathrm{~K}$ & $350 / 300 \mathrm{~K}$ & $*$ \\
Visual extinction & $2 \& 10 \mathrm{mag}$ & $2 \& 10 \mathrm{mag}$ & $2 \& 10 \mathrm{mag}$ & $2 \& 10 \mathrm{mag}$ & $*$ \\
External UV radiation intensity & $1 \mathrm{Habing}$ & $1000 \mathrm{Habing}$ & $1 \mathrm{Habing}$ & $1000 \mathrm{Habing}$ & $1 \mathrm{Habing}$ \\
Cosmic ray ionisation rate $(\zeta)$ & $1.3 \times 10^{-17} \mathrm{~s}^{-1}$ & $1.3 \times 10^{-17} \mathrm{~s}^{-1}$ & $1.3 \times 10^{-15} \mathrm{~s}^{-1}$ & $1.3 \times 10^{-15} \mathrm{~s}^{-1}$ & $1.3 \times 10^{-15} \mathrm{~s}^{-1} \mathrm{~s}$ \\
\hline
\end{tabular}

Notes. ${ }^{(*)}$ Here, the temperature in phase II and the visual extinction vary as the shock passes through the gas. ${ }^{(\dagger)}$ First value corresponds to $\mathrm{A}_{\mathrm{V}}=2 \mathrm{mag}$ and second to $\mathrm{A}_{\mathrm{V}}=10 \mathrm{mag}$.

region $(\mathrm{PDR})$ models can be used to qualitatively determine the origin of each molecule and give an indication of the relative contribution among the different gas components.

The UCL_CHEM was used to first simulate the formation of a dark core by collapsing an atomic diffuse gas from a density of $100 \mathrm{~cm}^{-3}$ to a final density of $10^{5} \mathrm{~cm}^{-3}$. During the collapse, atoms and molecules freeze onto the dust grains forming icy mantles. Both gas and surface chemistries are self-consistently computed. Once the gas is in dynamical equilibrium again, UCL_CHEM compiles the chemical evolution of the gas and the dust after a burst of star formation has occurred. While the temperature during the first phase of the modelling was kept to $10 \mathrm{~K}$, during the second phase it is increased to $100-350 \mathrm{~K}$ and the icy mantles are evaporated. The chemical evolution of the gas is then followed for $10^{7}$ years. In both phases of the UCL_CHEM model the chemical network is based on more than 2345 chemical reactions involving 205 species, of which 51 are surface species. The gas phase reactions were adopted from the UMIST data base (Millar et al. 1997; Le Teuff et al. 2000; Woodall et al. 2007). All the molecules detected so far in galactic hot cores (e.g. Blake et al. 1987; Millar \& Hatchell 1998; Hatchell et al. 1998; Fontani et al. 2007) have been included in this chemistry network, together with all the important and familiar molecular species that are known from many other studies to play a significant role in the chemistry of interstellar gas. The surface reactions included in this model are assumed to be mainly hydrogenation reactions, allowing chemical saturation where this is possible.

The adopted values for the free parameters of the UCL_CHEM model are listed in Table 4. Parameters not mentioned in this table have been kept to their standard (Milky Way) values. We used a final density of $n_{\mathrm{H}}=10^{5} \mathrm{~cm}^{-2}$ and a metallicity value of $z=1.056 z_{\odot}$, based on previous studies of NGC 1068 (e.g. Zaritsky et al. 1994; Tacconi et al. 1994; Kamenetzky et al. 2011). The initial elemental abundances $(\mathrm{C} / \mathrm{H}, \mathrm{O} / \mathrm{H}, \mathrm{N} / \mathrm{H}, \mathrm{S} / \mathrm{H}$, $\mathrm{He} / \mathrm{H}, \mathrm{Mg} / \mathrm{H}$, and $\mathrm{Si} / \mathrm{H}$ ) used in our study are those corresponding to extragalactic environments (as described in Bayet et al. 2008), scaled to the NGC 1068 metallicity.

To sample the likely co-existing conditions that molecules are experiencing in the nucleus of NGC 1068, we ran five models. The first four ( $a, b, c$, and $d$ ) allowed us to investigate the response of the chemistry to the changes in FUV radiation field and cosmic ray ionisation rate, $\zeta^{4}$. High cosmic ray ionisation rates can be used to qualitatively simulate XDR-like environments (see e.g. Bayet et al. 2009a). In fact, only a few species $\left(\mathrm{OH}^{+}, \mathrm{OH}, \mathrm{H}_{2} \mathrm{O}, \mathrm{H}_{3} \mathrm{O}^{+}\right)$show clear differences in abundances between regions pervaded by cosmic rays and X-rays (Meijerink et al. 2011). Since none of these molecules were observed in our

\footnotetext{
4 Models $a$ and $b$, characterised by a low $\zeta$, aim to explore how the chemistry changes as a function of the FUV intensity. Models $c$ and $d$, characterised by a high $\zeta$, aim to explore how the chemistry of an XDR-like environment changes as a function of FUV intensity.
}

survey, for the purpose of the modelling we assume that both enhanced $\zeta$ and X-rays leave similar fingerprints in the ISM.

Our models $c$ and $d$, which are characterised by a high $\zeta$ of 1000 times the canonical value of the Milky Way, aim to reproduce chemical conditions similar to the NGC 1068 nucleus. On the other hand, model $e$ aims to study the possible influence of $\mathrm{C}$-shocks, because it has recently been claimed that they may be affecting the gas in the CND of NGC 1068 (García-Burillo et al. 2010; Krips et al. 2011; Kamenetzky et al. 2011). To do this, we used the initial conditions of model $c$ and ran a version of UCL_CHEM coupled with the parametric shock model developed by Jiménez-Serra et al. (2008), which considers a plane-parallel C-Shock propagating with a velocity $v$ (we set $v=40 \mathrm{~km} \mathrm{~s}^{-1}$ ) through the ambient medium. Details of this version of UCL_CHEM can be found in Viti et al. (2011). The influence of shocks in the NGC 1068 nucleus is discussed further in Sect. 5.3.

Using the results of the models, we calculated the column densities of the seventeen species detected in our survey (carbon, oxygen, and sulphur isotopologues are not included) at a representative time of $10^{5} \mathrm{yr}$ (but note that chemistry has not necessarily reached steady state by then), using:

$N_{\text {mol }}=X_{\text {mol }} \times A_{\mathrm{V}} \times 1.6 \times 10^{21}$

where $X_{\mathrm{mol}}$ is the fractional abundance of the molecule, $A_{\mathrm{V}}$ the visual extinction, and $1.6 \times 10^{21} \mathrm{~cm}^{-2}$ the column density of hydrogen at a visual extinction of one magnitude. The formulation above simply gives an "on the spot" approximation of the column density. We set $N_{\text {mol }}=10^{12} \mathrm{~cm}^{-2}$ as the minimum theoretical column density to consider a species detectable. Below this value we do not take the results of the models into account. We include, however, some species that were not detected in our survey $\left(\mathrm{CH}_{3} \mathrm{CCH}, \mathrm{H}_{2} \mathrm{CO}, \mathrm{SO}_{2}, \mathrm{H}_{2} \mathrm{CS}\right)$, but whose response to UV fields, cosmic rays, and shocks may help us understand the differences in chemistry between AGNs and starburst galaxies.

\subsection{Origin of the molecules}

Disentangling the origin of extragalactic molecular emission is not trivial, because the same molecules may often arise from very different gas conditions (e.g. Bayet et al. 2008, 2009). For the purpose of this analysis, we considered a two-component model: molecular emission arising from the outer layers of molecular clouds, which are more exposed to external radiation fields that form PDRs; and emission arising from denser gas, closer to the centre of the clouds. These two gas components are represented in our model by visual extinctions of $A_{\mathrm{V}}=2 \mathrm{mag}$ (PDRs) and $10 \mathrm{mag}$ (dense gas), similar to previous studies (Bayet et al. 2008, 2009a; Meijerink \& Spaans 2005; Spaans \& Meijerink 2005). We note that above a certain critical visual extinction ( 2.5-3 mag, depending on the radiation field) the chemistry is no longer affected by UV radiation and therefore any $A_{\mathrm{V}}$ above that could have been chosen to represent the 
Table 5. Origin of the molecules according to each model.

\begin{tabular}{|c|c|c|c|c|}
\hline Molecule & Model $a$ & Model $b$ & Model $c$ & Model $d$ \\
\hline $\mathrm{CO}$ & $\mathrm{P} \& \mathrm{D}$ & $\bar{D}$ & P\& D & $\bar{D}$ \\
\hline $\mathrm{C}_{2} \mathrm{H}$ & P\& D & P\& D & P\& D & P\& D \\
\hline $\mathrm{CN}$ & P\& D & P\& D & P\& D & P\& D \\
\hline $\mathrm{HCN}$ & P\& D & D & P\& D & D \\
\hline $\mathrm{CS}$ & P\& D & D & P\& D & D \\
\hline $\mathrm{HCO}^{+}$ & - & - & P\& D & D \\
\hline $\mathrm{HNC}$ & P\& D & D & P\& D & D \\
\hline $\mathrm{HCO}$ & P\& D & - & P\& D & - \\
\hline $\mathrm{N}_{2} \mathrm{H}^{+}$ & - & - & P\& D & D \\
\hline $\mathrm{SiO}$ & D & D & P\& D & D \\
\hline $\mathrm{CH}_{3} \mathrm{CN}$ & D & D & P\& D & D \\
\hline NS & - & - & P\& D & D \\
\hline $\mathrm{H}_{2} \mathrm{CO}$ & D & D & P\& D & D \\
\hline $\mathrm{HC}_{3} \mathrm{~N}$ & D & - & - & - \\
\hline $\mathrm{CH}_{3} \mathrm{OH}$ & D & - & - & - \\
\hline SO & D & D & D & D \\
\hline $\mathrm{CH}_{3} \mathrm{CCH}$ & D & D & D & D \\
\hline $\mathrm{SO}_{2}$ & D & D & D & D \\
\hline $\mathrm{H}_{2} \mathrm{CS}$ & D & D & - & - \\
\hline $\mathrm{HOC}^{+}$ & - & - & - & - \\
\hline
\end{tabular}

Notes. $P$ stands for PDR emission, $D$ for dense gas emission (evaluated at visual extinctions of 2 mag and 10 mag respectively). $P \& D$ indicates that the molecule may arise from both molecular cloud regions. "-" means that the model give a column density below $10^{12} \mathrm{~cm}^{-2}$ (theoretical detectable limit), and thus the result is not taken into account.

denser, shielded gas component. As a result, increasing or decreasing the $A_{\mathrm{V}}$ would simply imply a variation in the estimated column densities by the same factor (see Eq. (1)).

In Table 5, we list all molecules and classify them as "tracers" of a particular gas component for each model. We found that $\mathrm{C}_{2} \mathrm{H}$ and $\mathrm{CN}$ always trace PDRs and dense gas independently of the strength of both UV fields and CRs (i.e. according to all the models). On the other hand, $\mathrm{CO}, \mathrm{HCN}, \mathrm{CS}, \mathrm{SiO}$, $\mathrm{CH}_{3} \mathrm{CN}, \mathrm{HNC}$, $\mathrm{SO}, \mathrm{CH}_{3} \mathrm{CCH}, \mathrm{H}_{2} \mathrm{CO}$, and $\mathrm{SO}_{2}$ are invariably found in dense gas regions. We note that many molecules can trace PDRs, as well as the denser, shielded regions, depending on the strength of UV fields and CRs adopted (Table 5), highlighting once more (e.g. Bayet et al. 2009) that one cannot easily isolate PDR-tracers as templates for modelling galaxies. These results generally agree with previous observational and theoretical studies (e.g. Huggins et al. 1984; Sternberg \& Dalgarno 1995; Bayet et al. 2008, 2009a; Aladro et al. 2011a).

\subsection{Influence of UV fields and cosmic rays on molecules}

Most molecular species are sensitive to the presence of external fields. We checked the variations in their column densities as a function of UV and CRs strengths (i.e. for the models $a$, $b, c$, and $d$ ). A summary of the findings is shown in Table 6 . While the column density of $\mathrm{HOC}^{+}$is enhanced by the presence of UV radiation, many other species are easily dissociated by UV fields in the external layers of the molecular clouds (such as $\mathrm{CO}$, $\mathrm{HCN}$, or NS). In contrast, we found that methanol $\left(\mathrm{CH}_{3} \mathrm{OH}\right)$ is clearly destroyed by cosmic rays, while the production of several other species is favoured by the presence of $\mathrm{CR}$ photons in the ISM (e.g. $\mathrm{SiO}, \mathrm{CN}$, or $\mathrm{N}_{2} \mathrm{H}^{+}$). Of particular interest is $\mathrm{SiO}$, commonly considered as a shock tracer, but whose enhanced abundance in NGC 1068 can also be related to high rates of X-rays (Usero et al. 2004; García-Burillo et al. 2010) and cosmic rays (this work). Finally, only $\mathrm{C}_{2} \mathrm{H}$ shows similar column densities (within one order of magnitude of variation) for all the models.
Table 6. Molecular trends with UV fields and cosmic rays.

\begin{tabular}{lc}
\hline \hline Easily dissociated by UV fields & $\mathrm{CO}, \mathrm{CS}, \mathrm{HCN}, \mathrm{CH}_{3} \mathrm{OH}$, \\
& $\mathrm{HCO}, \mathrm{SO}, \mathrm{NS}, \mathrm{HNC}^{\mathrm{SO}}$, \\
& $\mathrm{N}_{2} \mathrm{H}^{+}, \mathrm{SiO}, \mathrm{CH}_{3} \mathrm{CN}, \mathrm{H}_{2} \mathrm{CS}$, \\
& $\mathrm{H}_{2} \mathrm{CO}, \mathrm{HC}_{3} \mathrm{~N}, \mathrm{CH}_{3} \mathrm{CCH}$ \\
Enhanced by UV fields & $\mathrm{HOC}^{+}$, \\
Easily dissociated by cosmic rays & $\mathrm{CH}_{3} \mathrm{OH}, \mathrm{HC}_{3} \mathrm{~N}$ \\
Enhanced by cosmic rays & $\mathrm{CN}, \mathrm{HCN}^{+} \mathrm{HCO}^{+}, \mathrm{HNC}, \mathrm{SO}$, \\
Insensitive & $\mathrm{NS}, \mathrm{N}_{2} \mathrm{H}^{+}, \mathrm{SiO}_{2} \mathrm{SO}_{2}, \mathrm{HOC}^{+}$ \\
\hline
\end{tabular}

Our results agree well with those of Bayet et al. (2008), who also used the UCL_CHEM model to find suitable molecular tracers of hot cores under different physical conditions. In general, the trends of chemical abundances with respect to the $\zeta$ variations found by Bayet et al. (2008) are similar to what is shown in our Table 6, with few exceptions, where CS is the most contrasted species between both works. For this species Bayet et al. (2008) predicted a decrease in its abundance with increased $\zeta$, while our models predict a slight increase. This may be explained by the different physical parameters used in both works (other $\zeta$ strengths, initial and final densities, visual extinction, and final temperatures).

On the other hand, the comparison of our models with those that explored the PDR chemistry in a variety of extragalactic environments (Bayet et al. 2009a) leads to big discrepancies regarding the response of the molecular abundances as a function of $\zeta$ and FUV radiation fields. For example, Bayet et al. (2009a) deduce that $\mathrm{CN}$ and $\mathrm{HCO}^{+}$are not affected by the variations in $\zeta$, while our results predict enhancements of those species. Also, they find that $\mathrm{CO}, \mathrm{HNC}, \mathrm{HCN}$, and $\mathrm{H}_{2} \mathrm{CO}$ are not influenced by changes in the FUV radiation field, whereas our results indicate that the abundances of these molecules are reduced by at least one order of magnitude when the FUV field increases. These disagreements are mainly because Bayet et al. (2009a) used the UCL_PDR model where, unlike the UCL_CHEM code used here, the depletion of atoms and molecules onto grains, and the subsequent surface chemistry, is not included. In other words, our present modelling assumes that even the gas affected by UV photons has undergone sufficient processing involving gas-grain interactions.

\subsection{Comparison with the observations}

By considering a multi-component model we succeed in qualitatively reproducing the observed column densities of almost all the detected molecules in this survey within one order of magnitude, with $\mathrm{HOC}^{+}$the only exception. We consider this a good match, as the column densities have relevant uncertainties due to assuming LTE conditions. In general, the observations are better reproduced by the models that consider a high cosmic ray ionisation rate, but low FUV radiation field (model $c$ ) or high values of both parameters (model $d$ ). Once more, this result supports the use of high $\zeta$ rates in time- and depth-dependent chemical models as a good approximation of XDR-like environments.

However, as expected, the scenario depicted by the models reflects clear differences in the origin and timescales of the species, as well as highlights some degeneracies; nevertheless sometimes the same degeneracies can emphasise the prevalence of a particular energetic process. For example $\mathrm{CO}$, ubiquitously tracing gas at a wide range of densities, is well matched by all the models, yet models with a high and low radiation field match the observations better, as long as the cosmic ray ionisation rate is high. On the other hand, methanol is much better matched by a 
model where both radiation field and cosmic ray ionisation rates are low: we interpret this "mismatch" in physical conditions between $\mathrm{CO}$ and $\mathrm{CH}_{3} \mathrm{OH}$ as an indication that the two species, on average, arise from different gases; methanol clearly seem to arise from regions where the cosmic ray ionisation rate is close to standard, probably not representative of the average cosmic ray ionisation rate of the galaxy. This rate is, in fact, well known to be variable within our own galaxy (Dalgarno 2006), and it may well vary in NGC1068, too. On the other hand, the $\mathrm{HCO}^{+}$ observed abundance is only matched if the cosmic ray ionisation rate is high (i.e. Models $c$ and $d$ ), independently of the FUV field strength.

Unlike for cosmic rays, there are very few species that help us to determine the average UV field(s) strength in NGC1068. Of particular interest is $\mathrm{HCO}$, which seems to get closer to the observed value only in models where the UV field is low, while there is no molecule that needs a high radiation field in order to match the observations. While this does not exclude a high radiation field for this galaxy, especially near the nucleus, it seems unlikely that a rich chemistry comprised of species such as HCO would survive if the average radiation field were to be much higher than the canonical interstellar one.

On the other hand, $\mathrm{C}_{2} \mathrm{H}$ is better reproduced in a shocked environment, since $\mathrm{C}$-type shocks enhance its abundance up to the observational value. In contrast, $\mathrm{H}_{2} \mathrm{CO}$ seems to be destroyed by C-shocks. This, as well as strong UV fields, might be the reason for its non-detection in our survey. Apart from $\mathrm{C}_{2} \mathrm{H}$ and $\mathrm{H}_{2} \mathrm{CO}$, the presence of shocks does not greatly affect the abundances of our observed species. In particular we note that the $\mathrm{SiO}$ column density does not change if shocks are present, suggesting that its enhanced abundance is due to X-rays/cosmic rays gas processing instead of shocks. Although they might be present, our models therefore do not require the presence of strong shocks in the CND of NGC 1068. It is worth underlining here, however, that our models only include a treatment for C-type shocks: in these models, once the dense gas reaches its final pre-shocked density (i.e. at the end of Phase I), the gas and dust temperatures are already high enough $(250 \mathrm{~K})$ that many exothermic reactions are relatively efficient. The maximum temperature reached due to the shock is only a few hundred Kelvin higher than the initial temperature, and moreover it does not last for long ( $\sim 100-200$ years) before the gas starts cooling down. As a result, chemically there is not much difference between this scenario and the typical "hot core" scenario. The introduction of J-type shocks would significantly alter the chemistry.

Finally, it is interesting to note that our models show different recycling times among molecules. This behaviour seems to be related to the nature of the species rather than to a dependence on the radiation field and cosmic ray strength. For example, the column densities of some species, such as $\mathrm{CH}_{3} \mathrm{OH}$ and NS, are better matched at early times $\left(\sim 10^{3} \mathrm{yr}\right)$, since their abundances quickly drop at later times by at least three orders of magnitude. Similarly, the column densities of some important undetected species (e.g. $\mathrm{CH}_{3} \mathrm{CCH}$ ) drop dramatically below the detectable levels at later times (i.e. $\sim 10^{5} \mathrm{yr}$ ). On the other hand, $\mathrm{CO}, \mathrm{HCO}^{+}$, and $\mathrm{HOC}^{+}$always maintain constant values.

\section{Comparison with other galactic circumnuclear regions}

\subsection{Isotopic ratios}

For comparison purposes, Table 3 lists the isotopic line ratios obtained in the Galactic centre (GC) and the inner few hundred parsecs of the starburst galaxy M 82. As discussed in Sect. 4.3, our upper limits to the ${ }^{12} \mathrm{C} /{ }^{13} \mathrm{C}$ ratio in NGC 1068 are certainly affected by opacity effects. Also, selective photoionisation and chemical fractionation could be altering our carbon and oxygen isotopic ratios in NGC 1068. These effects might partially explain the isotopic differences among galaxies.

The most stringent lower limit to the ${ }^{12} \mathrm{C} /{ }^{13} \mathrm{C}$ ratio obtained in M 82 so far (>138, Martín et al. 2010) is more than two times higher than our best value in NGC 1068. However, we note that the $\mathrm{M} 82$ ratio was calculated from $\mathrm{C}_{2} \mathrm{H}$, while for $\mathrm{NGC} 1068$ we are using our $\mathrm{CN}$ results. Both molecules can be tracing different gas phases in the galaxies, so it is not surprising that they show distinct values. If we compare the carbon isotopic ratios of both galaxies using $\mathrm{CO}$ and $\mathrm{HCO}^{+}$(Henkel \& Mauersberger 1993), the results are similar.

On the other hand, the difference in carbon isotopic ratios between galaxy nuclei may also be related to the degree of processed material. ${ }^{12} \mathrm{C}$ and ${ }^{13} \mathrm{C}$ are products of primary and secondary nuclear processing in stars, respectively, and thus their ratio depends on the nucleosynthesis of stars, on stellar evolution, and hence on the star formation history of the galaxy. The stellar population age in the centre of NGC 1068 (200-300 Myr, Davies et al. 2007) is much older than that of M 82 ( 15 Myr, Konstantopoulos et al. 2009). Primary-to-secondary isotopic ratios are predicted to decrease with time, so a lower ${ }^{12} \mathrm{C} /{ }^{13} \mathrm{C}$ ratio (as well as ${ }^{16} \mathrm{O} /{ }^{18} \mathrm{O}$ ) is expected in NGC 1068 than in M 82. On the other hand, both the Galactic centre and the NGC 1068 nucleus have low carbon isotopic ratios as compared to M 82 (Table 3). This implies that both nuclei have a high chemical processing degree.

Our limit to the ${ }^{32} \mathrm{~S} /{ }^{34} \mathrm{~S}$ in NGC 1068 is a factor of four lower than the ratios in the GC and M 82. In the Milky Way, it was observed that ${ }^{32} \mathrm{~S} /{ }^{34} \mathrm{~S}$ does not strongly vary with time, although increases with the galacticentric distance, following the ${ }^{12} \mathrm{C} /{ }^{13} \mathrm{C}$ gradient of the galactic disk (Frerking et al. 1980; Wilson \& Rood 1994; Chin et al. 1996). Also, it was proposed that this ratio depends on the stellar population, so that environments with more massive stars and supernova explosions (the case for NGC 1068 as compared to the GC and M 82) have lower ${ }^{32} \mathrm{~S} /{ }^{34} \mathrm{~S}$.

\subsection{Molecular abundances}

M 82 and NGC 253 are the starburst galaxies with the best known chemical compositions so far. Like NGC 1068, both have been the targets of unbiased molecular line surveys toward their nuclei (Martín et al. 2006; Aladro et al. 2011b). The starburst in the centre of M 82 is old, with an average stellar population age of $\sim 10 \mathrm{Myr}$ (Konstantopoulos et al. 2009). This, together with a high supernova rate, creates strong UV fields, so that PDRs dominate its chemistry. On the other hand, NGC 253 is said to be in an earlier stage of starburst evolution (Martín et al. 2009; Aladro et al. 2011a), with younger stellar populations in its nucleus ( 6 Myr, Fernández-Ontiveros et al. 2009) where the PDRs, although present, do not characterise its ISM. Instead, the nucleus of NGC 253 show that large-scale shocks are the dominant process driving the molecular abundances.

Figure 3 shows the abundances of all species detected by single-dish $\mathrm{mm} / \mathrm{sub}-\mathrm{mm}$ telescopes in the three galaxies, as well as other species that were identified in both starburst galaxies but remain undetected in NGC 1068 (for them we used the upper limits to the column densities listed in Table 2). This figure aims to highlight the chemical differences between AGN-dominated and starburst-dominated nuclei. The fractional abundances were calculated with respect to $\mathrm{C}^{34} \mathrm{~S}$, which is significantly less 
M82 NGC253 NGC1068

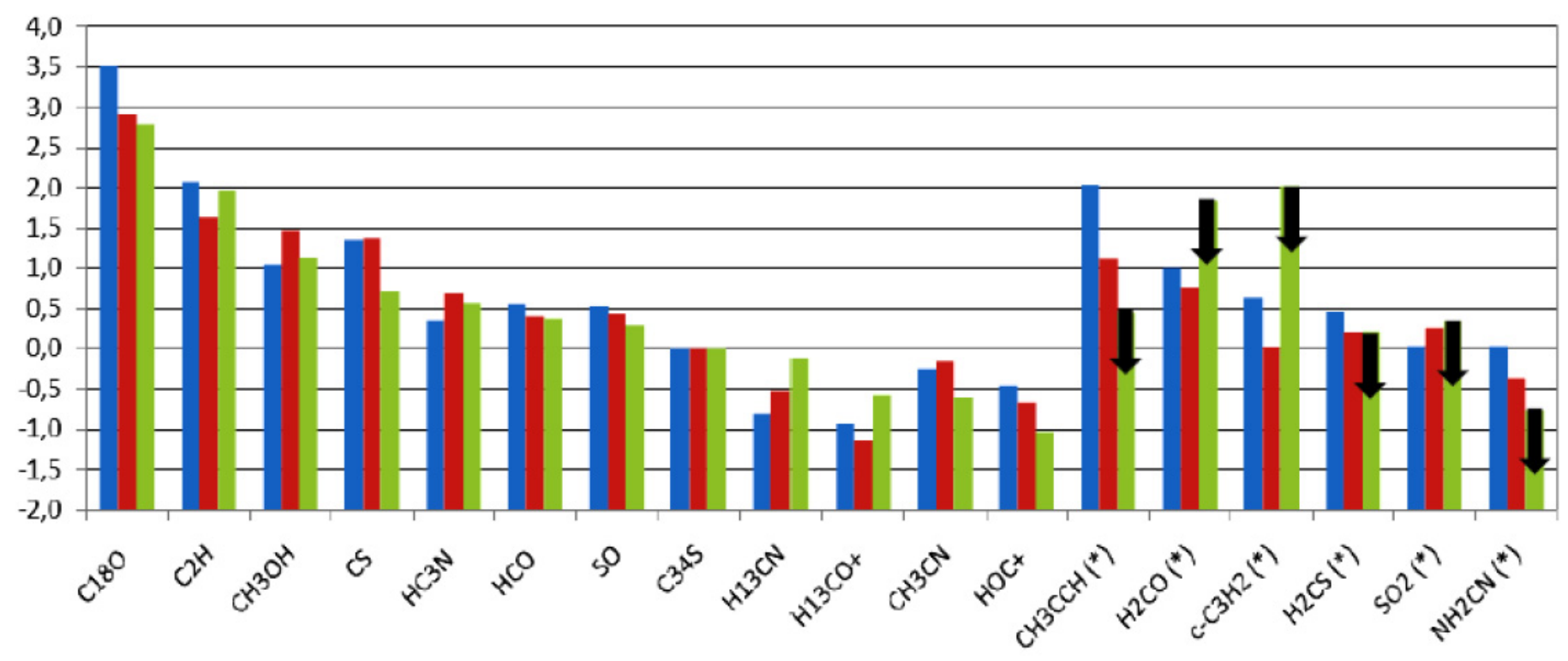

Fig. 3. Comparison of the fractional abundances among the two starbust galaxies M 82 (blue) and NGC 253 (red), and the AGN NGC 1068 (green). $Y$-axis is $\log _{10}\left(\mathrm{~N}_{\text {mol }} / \mathrm{N}_{\mathrm{C}^{34} \mathrm{~S}}\right)$. Molecules marked with an * were not detected in our NGC 1068 survey; arrows indicate their upper limits to the fractional abundances.

affected by opacity effects than CS, and whose abundance does not seem to be significantly affected by the type of nuclear activity (Martín et al. 2009).

We note that, while M 82 and NGC 253 are located at about the same distance (3.6 and $3.9 \mathrm{Mpc}$ respectively, Freedman et al. 1994; Karachentsev et al. 2003), NGC 1068 lies four times farther. However, while the comparison of the column densities in Fig. 3 would be biased by the different distances to the galaxies, this should not affect the comparison of the fractional abundances.

Among all the species compared, only the ratios $\mathrm{H}^{13} \mathrm{CN} / \mathrm{C}^{34} \mathrm{~S}$ and $\mathrm{H}^{13} \mathrm{CO}^{+} / \mathrm{C}^{34} \mathrm{~S}$ are higher in $\mathrm{NGC} 1068$ than in the starburst galaxies, being between two and five times more abundant in the AGN. Conversely, the fractional abundances of $\mathrm{C}_{2} \mathrm{H}, \mathrm{CH}_{3} \mathrm{OH}, \mathrm{HC}_{3} \mathrm{~N}, \mathrm{HCO}$, and $\mathrm{SO}$ show similar values in both types of nuclei, with differences within a factor of two among the galaxies. This suggests that these species are not especially affected by the different nuclear activities in starburst galaxies and AGN, as previously suggested by Nakajima et al. (2011). A third group is formed by those species whose fractional abundances are clearly higher in both M 82 and NGC 253 than in NGC 1068. Examples are $\mathrm{CH}_{3} \mathrm{CN} / \mathrm{C}^{34} \mathrm{~S}$ or $\mathrm{HOC}^{+} / \mathrm{C}^{34} \mathrm{~S}$, which show differences in the abundances between three and five times. It is remarkable that these molecules have very similar abundances in M 82 and NGC 253. However, the biggest contrasts are given by those molecules that have been clearly detected in both starburst galaxies, but not in our NGC 1068 survey. Examples are $\mathrm{CH}_{3} \mathrm{CCH}, \mathrm{H}_{2} \mathrm{CO}$, c- $\mathrm{C}_{3} \mathrm{H}_{2}$, $\mathrm{H}_{2} \mathrm{CS}, \mathrm{SO}_{2}$, and $\mathrm{NH}_{2} \mathrm{CN}$. We note that propyne $\left(\mathrm{CH}_{3} \mathrm{CCH}\right)$ and formaldehyde $\left(\mathrm{H}_{2} \mathrm{CO}\right)$ are abundant species in starburst galaxies (Mühle et al. 2007; Aladro et al. 2011a). Unlike $\mathrm{H}_{2} \mathrm{CO}$, we obtained a stringent upper limit to the $\mathrm{CH}_{3} \mathrm{CCH}$ column density in NGC 1068 (Table 2), which makes it the most contrasted species of our survey when comparing starburst galaxies and AGN (Fig. 3). We found that $\mathrm{CH}_{3} \mathrm{CCH} / \mathrm{C}^{34} \mathrm{~S}$ is $\sim 40$ and $\sim 5$ times more abundant in M 82 and NGC 253, respectively, than in NGC 1068 . This strong variation in the
$\mathrm{CH}_{3} \mathrm{CCH}$ fractional abundances is likely to be intrinsically related to the different nuclear activities in galaxies, rather than being due to other factors, such as different opacities (due to its K-ladder structure the transitions of this molecule are expected to be optically thin), or kinetic temperature of the gas (as we do not find a strong (anti-) correlation of its abundances with the $T_{\text {kin }}$ of the three galaxies).

\section{Conclusions}

We used the IRAM-30 m telescope to carry out a spectral line survey (from $86.2 \mathrm{GHz}$ to $115.6 \mathrm{GHz}$ ) towards the nucleus of the AGN galaxy NGC 1068. We detected a total of 35 spectral lines corresponding to 17 molecular species. Seven molecules, or their ${ }^{13} \mathrm{C}$ isotopologues, are detected for the first time in this galaxy, namely $\mathrm{HC}_{3} \mathrm{~N}, \mathrm{SO}, \mathrm{N}_{2} \mathrm{H}^{+}, \mathrm{CH}_{3} \mathrm{CN}, \mathrm{NS},{ }^{13} \mathrm{CN}$, and $\mathrm{HN}^{13} \mathrm{C}$. We note that some molecules that are abundant in other galaxies, such as $\mathrm{H}_{2} \mathrm{CO}$ or $\mathrm{CH}_{3} \mathrm{CCH}$, are not detected in NGC 1068 . We calculated the column densities of the identified species by assuming local thermodynamic equilibrium and optically thin emission. However, some intense lines, such as $J-J^{\prime}=1-0$ $\mathrm{CO}, \mathrm{HCO}^{+}, \mathrm{HCN}$, or $\mathrm{HNC}$, are affected by optical thickness (and might also be altered by chemical fractionation and isotopeselective photoionisation effects), so their column densities are lower limits to the actual values.

Using several lines from our survey we obtained the isotopic abundance ratios ${ }^{12} \mathrm{C} /{ }^{13} \mathrm{C}=49,{ }^{16} \mathrm{O} /{ }^{18} \mathrm{O} \geq 177$, and ${ }^{32} \mathrm{~S} /{ }^{34} \mathrm{~S}=5$ for NGC 1068. These isotopic ratios significantly differ from those of the Galactic centre and starburst galaxies. Apart from the fact that the opacities of the transitions change from source to source, this reflects the different star formation histories of the galaxies.

We ran chemical models to determine the origin of the species detected in our survey, as well as to test the influence of UV fields, cosmic rays, and shocks on their abundances. We used a high rate of cosmic ray ionisation rate to qualitatively simulate an XDR-like environment. We found that the assumption 
of some molecules being PDR tracers or dense gas tracers should be done carefully, and only if the physical conditions of the gas are known, since under certain circumstances a PDR tracer may be also arising from dense gas regions and vice versa. Our models indicate that a high cosmic ray ionisation rate is present in the nucleus of NGC 1068, albeit a combination of high cosmic ray ionisation rates and UV fields may also explain the observed abundances in the galaxy. On the contrary, shocks do not seem to be necessary to explain most of the molecular abundances. The chemical models applied to NGC 1068 predict that while $\mathrm{UV}$ radiation easily dissociate a large number of molecules (e.g. $\mathrm{HCN}, \mathrm{CH}_{3} \mathrm{OH}, \mathrm{HNC}$, or $\mathrm{CH}_{3} \mathrm{CCH}$ ), cosmic rays enhance the abundances of a good number of others, such as $\mathrm{CN}, \mathrm{SiO}, \mathrm{N}_{2} \mathrm{H}^{+}$, NS, or HCN.

We aimed to find the molecules that are more sensitive to the different power sources of galaxies nuclei (starbursts and active galactic nuclei). We carried out a detailed comparison of the chemical composition of the ISM in the NGC 1068 nucleus and those of the two starbursts galaxies M 82 and NGC 253. Examples of differing ratios are $\mathrm{H}^{13} \mathrm{CN} / \mathrm{C}^{34} \mathrm{~S}$, which is $\sim 3-5$ times more abundant in NGC 1068 than in starburst galaxies, and $\mathrm{CH}_{3} \mathrm{CCH} / \mathrm{C}^{34} \mathrm{~S}$, which is, at least, 5-40 times more abundant in starburst galaxies than in NGC 1068.

Acknowledgements. We thank the IRAM staff for their help with the observations. This work was supported by the Science \& Technology Facilities Council [ST/F501761/1] and also the Spanish Ministerio de Ciencia e Innovación under the projects ESP2007-65812-C02-01 and AYA 2010-21697-C05-01. S.M. acknowledges the co-funding of this work under the Marie Curie Actions of the European Commission (FP7-COFUND).

\section{References}

Aladro, R., Martín-Pintado, J., Martín, S., Mauersberger, R., \& Bayet, E. 2011a, A\&A, 525, A89

Aladro, R., Martín, S., Martín-Pintado, J., et al. 2011b, A\&A, 535, A84

Ao, Y., Henkel, C., Braatz, J. A., et al. 2011, A\&A, 529, A154

Bayet, E., Viti, S., Williams, D. A., \& Rawlings, J. M. C. 2008, ApJ, 676, 978

Bayet, E., Viti, S., Williams, D. A., Rawlings, J. M. C., \& Bell, T. 2009a, ApJ, 696, 1466

Bayet, E., Aladro, R., Martín, S., Viti, S., \& Martín-Pintado, J. 2009b, ApJ, 707, 126

Blake, G. A., Sutton, E. C., Masson, C. R., \& Phillips, T. G. 1987, ApJ, 315, 621 Bland-Hawthorn, J., Gallimore, J. F., Tacconi, L. J., et al. 1997, Ap\&SS, 248, 9 Chin, Y.-N., Henkel, C., Whiteoak, J. B., Langer, N., \& Churchwell, E. B. 1996, A\&A, 305, 960

Chu, Y.-H., \& Watson, W. D. 1983, ApJ, 267, 151

Dalgarno, A. 2006, Proc. of the National Academy of Science, 103, 12269

Davies, R. I., Sánchez, F. M., Genzel, R., et al. 2007, ApJ, 671, 1388

Fernández-Ontiveros, J. A., Prieto, M. A., \& Acosta-Pulido, J. A. 2009, MNRAS, 392, L16

Fontani, F., Pascucci, I., Caselli, P., et al. 2007, A\&A, 470, 639

Freedman, W. L., Hughes, S. M., Madore, B. F., et al. 1994, ApJ, 427, 628

Frerking, M. A., Wilson, R. W., Linke, R. A., \& Wannier, P. G. 1980, ApJ, 240, 65

García-Burillo, S., Usero, A., Fuente, A., et al. 2010, A\&A, 519, A2

Glassgold, A. E., Huggins, P. J., \& Langer, W. D. 1985, ApJ, 290, 615

Goldsmith, P. F., \& Langer, W. D. 1999, ApJ, 517, 209
Hatchell, J., Thompson, M. A., Millar, T. J., \& MacDonald, G. H. 1998, A\&AS, 133,29

Helfer, T. T., \& Blitz, L. 1995, ApJ, 450, 90

Henkel, C., \& Mauersberger, R. 1993, A\&A, 274, 730

Huggins, P. J., Glassgold, A. E., \& Morris, M. 1984, ApJ, 279, 284

Israel, F. P. 2009, A\&A, 493, 525

Jackson, J. M., Paglione, T. A. D., Ishizuki, S., \& Nguyen-Q-Rieu 1993, ApJ, 418, L13

Jaffe, W., Meisenheimer, K., Röttgering, H. J. A., et al. 2004, Nature, 429, 47

Jiménez-Serra, I., Caselli, P., Martín-Pintado, J., \& Hartquist, T. W. 2008, A\&A, 482,549

Kamenetzky, J., Glenn, J., Maloney, P. R., et al. 2011, ApJ, 731, 83

Karachentsev, I. D., Grebel, E. K., Sharina, M. E., et al. 2003, A\&A, 404, 93

Kohno, K., Matsushita, S., Vila-Vilaró, B., et al. 2001, The Central Kiloparsec of Starbursts and AGN: The La Palma Connection, 249, 672

Konstantopoulos, I. S., Bastian, N., Smith, L. J., et al. 2009, ApJ, 701, 1015

Krips, M., Neri, R., García-Burillo, S., et al. 2008, ApJ, 677, 262

Krips, M., Martín, S., Eckart, A., et al. 2011, ApJ, 736, 37

Le Teuff, Y. H., Millar, T. J., \& Markwick, A. J. 2000, A\&AS, 146, 157

Lovas, F. J. 1992, J. Phys. Chem. Ref. Data, 21, 181

Maloney, P. R., Hollenbach, D. J., \& Tielens, A. G. G. M. 1996, ApJ, 466, 561

Martín, S., Martín-Pintado, J., \& Mauersberger, R. 2006, A\&A, 450, L13

Martín, S., Martín-Pintado, J., \& Mauersberger, R. 2009, ApJ, 694, 610

Martín, S., Aladro, R., Martín-Pintado, J., \& Mauersberger, R. 2010, A\&A, 522, A62

Meijerink, R., \& Spaans, M. 2005, A\&A, 436, 397

Meijerink, R., Spaans, M., Loenen, A. F., \& van der Werf, P. P. 2011, A\&A, 525, A119

Millar, T. J., \& Hatchell, J. 1998, Faraday Discussions, 109, 15

Millar, T. J., MacDonald, G. H., \& Gibb, A. G. 1997, A\&A, 325, 1163

Mühle, S., Seaquist, E. R., \& Henkel, C. 2007, ApJ, 671, 1579

Müller, H. S. P., Thorwirth, S., Roth, D. A., \& Winnewisser, G. 2001, A\&A, 370, L49

Müller, H. S. P., Schlöder, F., Stutzki, J., \& Winnewisser, G. 2005, J. Mol. Struct., 742,215

Müller Sánchez, F., Davies, R. I., Genzel, R., et al. 2009, ApJ, 691, 749

Nakajima, T., Takano, S., Kohno, K., \& Inoue, H. 2011, ApJ, 728, L38

Papadopoulos, P. P., \& Seaquist, E. R. 1999, ApJ, 516, 114

Papadopoulos, P. P., Seaquist, E. R., \& Scoville, N. Z. 1996, ApJ, 465, 173

Penzias, A. A., \& Burrus, C. A. 1973, ARA\&A, 11, 51

Pérez-Beaupuits, J. P., Spaans, M., van der Tak, F. F. S., et al. 2009, A\&A, 503, 459

Pickett, H. M., Poynter, I. R. L., Cohen, E. A., et al. 1998, J. Quant. Spect. Rad. Transf., 60, 883

Sakai, N., Saruwatari, O., Sakai, T., Takano, S., \& Yamamoto, S. 2010, A\&A, 512, A31

Schinnerer, E., Eckart, A., Tacconi, L. J., Genzel, R., \& Downes, D. 2000, ApJ, 533,850

Spaans, M., \& Meijerink, R. 2005, Ap\&SS, 295, 239

Sternberg, A., \& Dalgarno, A. 1995, ApJS, 99, 565

Sternberg, A., Genzel, R., \& Tacconi, L. 1994, ApJ, 436, L131

Tacconi, L. J., Genzel, R., Blietz, M., et al. 1994, ApJ, 426, L77

Telesco, C. M., \& Harper, D. A. 1980, ApJ, 235, 392

Usero, A., García-Burillo, S., Fuente, A., Martín-Pintado, J., \& Rodríguez-Fernández, N. J. 2004, A\&A, 419, 897

Viti, S., \& Williams, D. A. 1999, MNRAS, 305, 755

Viti, S., Collings, M. P., Dever, J. W., McCoustra, M. R. S., \& Williams, D. A. 2004, MNRAS, 354, 1141

Viti, S., Jimenez-Serra, I., Yates, J. A., et al. 2011, ApJ, 740, L3

Wannier, P. G. 1980, ARA\&A, 18, 399

Wilson, T. L., \& Rood, R. 1994, ARA\&A, 32, 191

Woodall, J., Agúndez, M., Markwick-Kemper, A. J., \& Millar, T. J. 2007, A\&A, 466, 1197

Woods, P. M., \& Willacy, K. 2009, ApJ, 693, 1360

Young, J. S., \& Sanders, D. B. 1986, ApJ, 302, 680

Zaritsky, D., Kennicutt, R. C., Jr., \& Huchra, J. P. 1994, ApJ, 420, 87 


\section{Appendix A: Comments on individual molecules}

We have detected seventeen molecular species, and seven carbon, sulphur, and oxygen isotopic substitutions in the $3 \mathrm{~mm}$ atmospheric window, between $86.2 \mathrm{GHz}$ and $115.6 \mathrm{GHz}$. Here, we detail each observed transition, the Gaussian profile-fitting, cases of blended lines, and other special cases. To identify the molecules, we started from the premise that only the transitions having $E_{\text {low }}<100 \mathrm{~cm}^{-1}$ and $\log \left(\int T_{\mathrm{MB}} \mathrm{d} v\right)>-6 \mathrm{~nm}^{2} \mathrm{MHz}$ contribute to the observed lines. After checking this initial condition, we ensured that the spectral features that do not fulfil these conditions can be considered negligible.

NGC 1068 has a heliocentric systemic velocity of $v_{\text {sys }}=$ $1137 \mathrm{~km} \mathrm{~s}^{-1}$ (value taken from NASA/IPAC Extragalactic Database - NED). Based on the values obtained from the Gaussian fits, we have chosen an average value of $1100 \mathrm{~km} \mathrm{~s}^{-1}$ for fixing this parameter when needed. We found two different line widths for the detected molecules in NGC 1068. In general, strong lines have values in the range $F W H M \sim$ [230-260] $\mathrm{km} \mathrm{s}^{-1}$, while faint lines have line widths of about $\sim[120-190] \mathrm{km} \mathrm{s}^{-1}$. The cases where it was necessary to fix this parameter are discussed below, since the value depends on each species.

- Hydrogen cyanide - HCN and $H^{13} C N$. We detected the $\mathrm{H}^{13} \mathrm{CN}(1-0)$ transition at $86.3 \mathrm{GHz}$. It is formed by three hyperfine spectral features, although the structure of the line is not resolved owing the broad line widths of NGC 1068. To take the contribution of each hyperfine feature into account we performed a synthetic Gaussian fit (details about this method can be seen in Martín et al. 2010; Aladro et al. 2011b). The resulting values, shown in Table B.1, correspond to the main component of the group. This $\mathrm{HCN}$ carbon isotopologue is detected for the first time in this galaxy.

$\mathrm{HCN}(1-0)$ is identified at $88.6 \mathrm{GHz}$. In a similar way as for $\mathrm{H}^{13} \mathrm{CN}$, we fitted a synthetic Gaussian profile leaving the linewidth and line position free.

- Hydrogen isocyanide - HNC and $H N^{13} C$. One strong line of hydrogen isocyanide was detected at $90.7 \mathrm{GHz}$. The telescope beam possibly picked up some emission coming from the starburst ring that surrounds the CND of NGC 1068. We complemented this transition with the HNC (4-3) line observed by Pérez-Beaupuits et al. (2009).

The $\mathrm{HN}^{13} \mathrm{C}(1-0)$ transition at $87.1 \mathrm{GHz}$ has been tentatively detected for the first time in this galaxy. Because it is quite faint, the integrated intensity resulting from the Gaussian fit is highly affected by the baseline. This is reflected in the column density derived from the rotational diagram, which has a high associated error.

- Ethynyl- $\mathrm{C}_{2} \mathrm{H}$. $\mathrm{C}_{2} \mathrm{H}(1-0)$, formed by six hyperfine features, is detected at $87.3 \mathrm{GHz}$. We performed a synthetic Gaussian fit to calculate the contribution of each feature. Because we only have one detected transition of this species, we calculated its column density by fixing the rotational temperature to $10 \pm 5 \mathrm{~K}$. This is the fourth most abundant molecule after $\mathrm{CO}$ and its carbon and oxygen isotopologues.

- Isocyanic acid - HNCO. Four groups of transitions were detected at $87.9 \mathrm{GHz}, 109.5 \mathrm{GHz}, 109.9 \mathrm{GHz}$, and $110.3 \mathrm{GHz}$. They are composed of five features each. The line at $109.9 \mathrm{GHz}$ is seen as a bump at the higher frequencies of $\mathrm{C}^{18} \mathrm{O}$, although both can be easily separated. The transition at $110.3 \mathrm{GHz}$ is very close to ${ }^{13} \mathrm{CO}$ and blended with $\mathrm{CH}_{3} \mathrm{CN}\left(6_{4,0}-5_{4,0}\right)$. We performed a synthetic Gaussian fit to all these lines, including two other HNCO transitions that are too faint to be detected, but that fulfil the

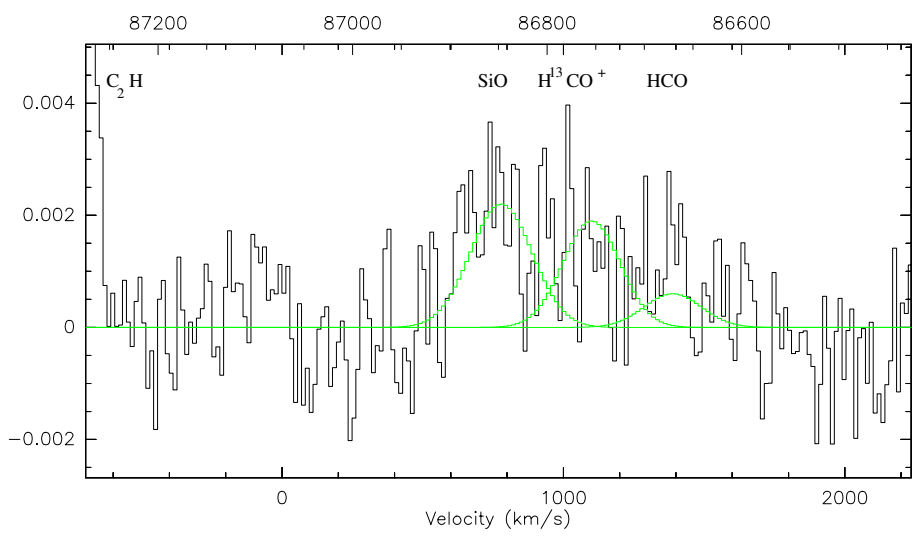

Fig. A.1. Gaussian fits to the blended lines of $\mathrm{H}^{13} \mathrm{CO}^{+}(1-0)$, $\operatorname{HCO}\left(1_{1,0}-0_{1,0}\right)$, and $\mathrm{SiO}(2-1)$. See more details in the text.

conditions mentioned at the beginning of this appendix $\left(E_{\text {low }}<100 \mathrm{~cm}^{-1}\right.$ and $\left.\log \left(\int T_{\mathrm{MB}} \mathrm{d} v\right)>-6 \mathrm{~nm}^{2} \mathrm{MHz}\right)$. In the Gaussian fitting we fixed the linewidth to $230 \mathrm{~km} \mathrm{~s}^{-1}$ and the line position to $1100 \mathrm{~km} \mathrm{~s}^{-1}$. The integrated area of our $\operatorname{HNCO}\left(5_{0,5}-4_{0,4}\right)$ line is consistent with the values obtained by Martín et al. (2009). However, from the Boltzmann diagram we obtain a rotational temperature of $T_{\text {rot }} \sim 30 \mathrm{~K}$, which is almost five times higher than the one obtained by Martín et al. (2009). This difference is due to the transitions with the higher energy levels we included.

- Oxomethylium - $\mathrm{HCO}^{+}$and $\mathrm{H}^{13} \mathrm{CO}^{+}$. The strong line of $\mathrm{HCO}^{+}(1-0)$ at $89.2 \mathrm{GHz}$ is the only detected transition in our line survey, and shows some emission coming from the starburst ring. We also used the $\mathrm{HCO}^{+}(3-2)$ line observed at $267.6 \mathrm{GHz}$ by Krips et al. (2008). The $\mathrm{H}^{13} \mathrm{CO}^{+}(1-0)$ line is detected at $86.8 \mathrm{GHz}$, although it is blended with $\operatorname{HCO}\left(1_{1,0}-0_{1,0}\right)$ and $\mathrm{SiO}(2-1)$. To disentangle the contribution of each molecule, we performed synthetic Gaussian fits to the three lines. We first fitted $\mathrm{H}^{13} \mathrm{CO}^{+}(1-0)$ by fixing the position to $1100 \mathrm{~km} \mathrm{~s}^{-1}$ and the linewidth to $240 \mathrm{~km} \mathrm{~s}^{-1}$. Then, we fitted the two other molecules (see Fig. A.1).

- Oxomethyl - HCO. Only one molecular transition of this species has been detected during the survey. It is blended with $\mathrm{H}^{13} \mathrm{CO}^{+}(1-0)$ and $\mathrm{SiO}(2-1)$ (see Figs. 2 and A.1). $\mathrm{HCO}\left(1_{1,0}-0_{1,0}\right)$ is located at $86.7 \mathrm{GHz}$, and contains four features. After subtracting the oxomethylium Gaussian fit, we over-fitted a Gaussian profile to the HCO features with a fixed position of $1100 \mathrm{~km} \mathrm{~s}^{-1}$ and a linewidth of $240 \mathrm{~km} \mathrm{~s}^{-1}$.

- Silicon monoxide - SiO. As discussed before, $\mathrm{SiO}(2-1)$ at $86.8 \mathrm{GHz}$ is blended with $\mathrm{H}^{13} \mathrm{CO}^{+}(1-0)$ and $\operatorname{HCO}\left(1_{1,0}-0_{1,0}\right)$. After subtracting the Gaussian fits of the other two molecules, we fitted another synthetic Gaussian profile to the residuals, fixing only the position to $1100 \mathrm{~km} \mathrm{~s}^{-1}$ (see Fig. A.1). This is the strongest molecule of the three blended lines.

- Methyl cyanide - $\mathrm{CH}_{3} \mathrm{CN}$. This symmetric top molecule is detected for the first time in NGC 1068. The two $\mathrm{CH}_{3} \mathrm{CN}\left(5_{k}-4_{k}\right)$ and $\mathrm{CH}_{3} \mathrm{CN}\left(6_{k}-5_{k}\right)$ transitions at $92.0 \mathrm{GHz}$ and $110.4 \mathrm{GHz}$ lie inside our frequency coverage. Each of them are formed by 25 spectral features with the quantum number $k$ going from 0 to $J-1$. Although faint, both lines are strong enough to be detected with an $S / N \geq 3$. The transition at $110.4 \mathrm{GHz}$ is blended with $\operatorname{HNCO}\left(5_{1,4}-4_{1,3}\right)$. We fitted a synthetic Gaussian profile to both lines, fixing the line position of $\mathrm{CH}_{3} \mathrm{CN}$ to $1100 \mathrm{~km} \mathrm{~s}^{-1}$ and the linewidth to $150 \mathrm{~km} \mathrm{~s}^{-1}$ (based on the non-blended line). Its rotational 
temperature is similar in NGC $1068\left(T_{\text {rot }} \sim 29 \mathrm{~K}\right)$ and M 82 $\left(T_{\text {rot }} \sim 33 \mathrm{~K}\right)$.

- Sulphur monoxide - SO. Three transitions of sulphur monoxide were detected for the first time in NGC 1068 at $99.3 \mathrm{GHz}$, 100.0 GHz, and 109.252 GHz. The first one was not blended, and its Gaussian parameters have been used to fit the other two. The line is narrow, $136 \mathrm{~km} \mathrm{~s}^{-1}$. The other two lines are blended with $\mathrm{HC}_{3} \mathrm{~N}(11-10)$ and $\mathrm{HC}_{3} \mathrm{~N}(12-11)$, respectively. The contribution of SO was firstly subtracted, and then we fitted cyanoacetylene to the residuals. The $\mathrm{SO}\left(5_{4}-4_{4}\right)$ at 100.0 GHz does not fit well in the rotational diagram, so the values we show in Table 1 do not take this transition into account.

- Hydroxymethylidynium - HOC ${ }^{+}$. We detected the $\mathrm{HOC}^{+}(1-0)$ transition at $89.5 \mathrm{GHz}$. Its peak intensity temperature is slightly above the noise, so we consider it as a tentative detection. This line shows a very narrow linewidth, of about $128 \mathrm{~km} \mathrm{~s}^{-1}$. Fixing the rotational temperature to $10 \pm 5 \mathrm{~K}$, we obtained the lowest column density of the molecular survey, with $N_{\mathrm{HOC}^{+}}=(1.1 \pm 0.6) \times 10^{13} \mathrm{~cm}^{-2}$.

- Cyanoacetylene - $H C_{3} N$. This molecule is detected for the first time in $\mathrm{NGC} 1068$. We identified $\mathrm{HC}_{3} \mathrm{~N}(10-9)$ at 91.0 GHz, $\mathrm{HC}_{3} \mathrm{~N}(11-10)$ at $100.1 \mathrm{GHz}$, and $\mathrm{HC}_{3} \mathrm{~N}(12-11)$ at $109.2 \mathrm{GHz}$. The transition at $91.0 \mathrm{GHz}$ was not blended, so we used its Gaussian parameters to fix the other two, which were blended with SO. For those lines, we first subtracted the sulphur monoxide features (as explained before), and then fitted two synthetic Gaussian profiles to the residuals. The $\mathrm{HC}_{3} \mathrm{~N}$ rotational temperature in NGC $1068(7.3 \pm 1.4 \mathrm{~K})$ is significantly lower than the ones found for M 82 and NGC 253 ( $25 \mathrm{~K}$ and $\sim[12-73] \mathrm{K}$, respectively, Aladro et al. 2011a).

- Diazenylium $-\mathrm{N}_{2} \mathrm{H}^{+}$. This is the first time that this species is detected in NGC 1068. Only the $\mathrm{N}_{2} \mathrm{H}^{+}(1-0)$ transition, at 93.2 GHz, lies inside our survey. It is formed by three features, so we performed a synthetic Gaussian fit in order to calculate the contribution of each one. We fixed the rotational temperature to $10 \pm 5 \mathrm{~K}$ to calculate its column density.

- Carbon monosulfide - CS and $C^{34} S$. We detected the $\mathrm{CS}(2-1)$ line, which is blended with ${ }^{13} \mathrm{CO}(1-0)$ coming from the image band. Since these two are the only transitions of these molecules detected in our survey, it is not possible to disentangle the contribution of each one. Thus, no Gaussian fit was done. Nevertheless, we used the CS (3-2), CS (5-4), and CS (7-6) lines observed by Bayet et al. (2009b) to do the rotation diagram. Two gas components with different temperatures and column densities are seen.

$\mathrm{C}^{34} \mathrm{~S}(2-1)$ was detected at $96.4 \mathrm{GHz}$. This transition was complemented with the $\mathrm{C}^{34} \mathrm{~S}(3-2)$ line observed by Martín et al. (2009). The rotational temperature is one of the lowest obtained in the survey, with only $\sim 4 \mathrm{~K}$.

- Carbon monoxide - CO, $C^{18} \mathrm{O}$, and ${ }^{13} \mathrm{CO}$. $\mathrm{CO}(1-0)$, at $115.3 \mathrm{GHz}$, was blended with NS $\left(3_{1}-2_{1}\right)$, but since NS was, comparatively speaking, very faint, we assumed that its contribution is negligible. Since the emission of $\mathrm{CO}(1-0)$ is quite spread in the nucleus of NGC 1068, it clearly shows a deviation from a Gaussian profile, which points to the complex gas dynamics in that region, as well as a possible contribution by the starburst ring emission. We fitted a three Gaussian profile, which is shown in Fig. A.2.

We also used the $\mathrm{CO}(2-1), \mathrm{CO}(3-2)$, and $\mathrm{CO}(4-3)$ lines from Israel (2009). Like CS, CO shows two components in

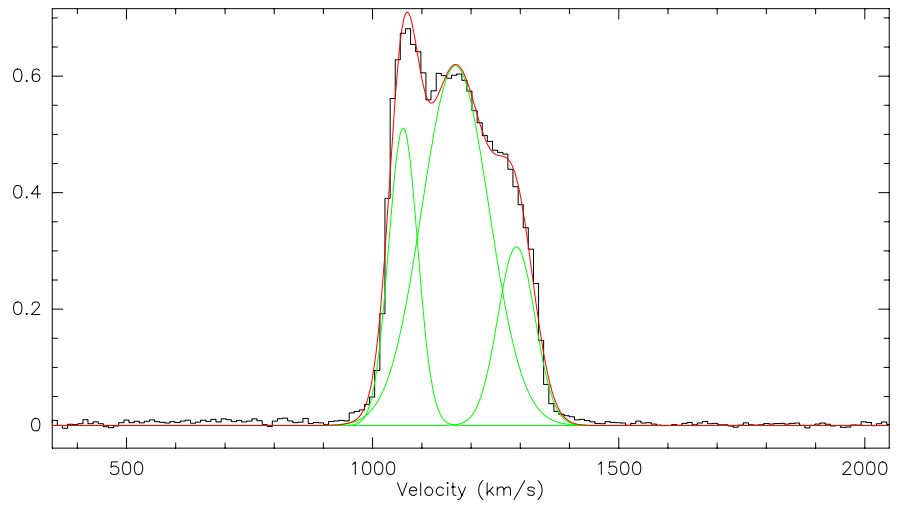

Fig. A.2. Line profile of the $\mathrm{CO}(1-0)$ line. $Y$-axis is on a $T_{\mathrm{mb}}$ scale.

the rotation diagram. Even though $\mathrm{CO}(1-0)$ is the strongest feature in the survey, it is not seen in the image band. This is due to the variable rejection along the $3 \mathrm{~mm}$ band.

${ }^{13} \mathrm{CO}(1-0)$ was detected at $110.2 \mathrm{GHz}$. Similarly to ${ }^{12} \mathrm{CO}(1-0)$, this transition showed a deviation from a Gaussian profile. It was complemented with the ${ }^{13} \mathrm{CO}(2-1)$ and ${ }^{13} \mathrm{CO}(3-2)$ lines observed by Israel (2009). Two different components are also seen in the Boltzmann diagram. As discussed before, ${ }^{13} \mathrm{CO}(1-0)$ does appear in the image band, blended with CS (2-1).

$\mathrm{C}^{18} \mathrm{O}(1-0)$ appears at $109.8 \mathrm{GHz}$. It was not blended, but it showed a bump at the higher frequencies due to the proximity of an HNCO transition. To avoid the isocyanic acid contamination, we fixed the linewidth to $230 \mathrm{~km} \mathrm{~s}^{-1}$ and then fitted three Gaussians, which accounts for the line profile better. We also used the $\mathrm{C}^{18} \mathrm{O}(2-1)$ line observed by Martín et al. (2009) for complementing our observations. The rotational temperature is the lowest one among all the molecules detected $\left(T_{\text {rot }}=3.3 \pm 0.1 \mathrm{~K}\right)$.

- Cyanogen - CN. Two separated lines of cyanogen were identified at $113.2 \mathrm{GHz}\left(\mathrm{CN}\left(1_{0,1}-0_{0,1}\right)\right)$ and $113.5 \mathrm{GHz}$ (CN $\left.\left(1_{0,2}-0_{0,1}\right)\right)$. The first one is formed by four different features, while the second one has five. We fitted a synthetic Gaussian profile to both lines. We included other CN transitions observed by Pérez-Beaupuits et al. (2009). We also detected two multi-feature lines of ${ }^{13} \mathrm{CN}$ at $108.4 \mathrm{GHz}$ and $108.6 \mathrm{GHz}$.

- Methanol - $\mathrm{CH}_{3} \mathrm{OH}$. Only the $\mathrm{CH}_{3} \mathrm{OH}\left(2_{k}-1_{k}\right)$ line is seen at $96.7 \mathrm{GHz}$, which is composed of four features with the quantum number $k$ varying between $-1,0,1$. However, we calculated the integrated intensities of other transitions that lie inside our frequency range, which fulfil the conditions imposed on the energy and logarithm of the integrated intensities, as explained at the beginning of this appendix. Considering all these features (thirteen in total), we performed a synthetic Gaussian fit.

- Nitrogen monosulphide - NS. We detected the NS $\left(3_{1}-2_{1}\right)$ transition in our survey. It is split into two separated multilines with six features each. The first one is blended with $\mathrm{CO}(1-0)$, while the second one is located at higher frequencies, so that it can be easily separated from $\mathrm{CO}$. We first subtracted the $\mathrm{CO}$ contribution, and then fitted a synthetic Gaussian profile to all the NS features. We assumed $T_{\text {rot }}=10 \pm 5 \mathrm{~K}$ to calculate the column density. 
R. Aladro et al.: A $\lambda=3 \mathrm{~mm}$ molecular line survey of NGC 1068
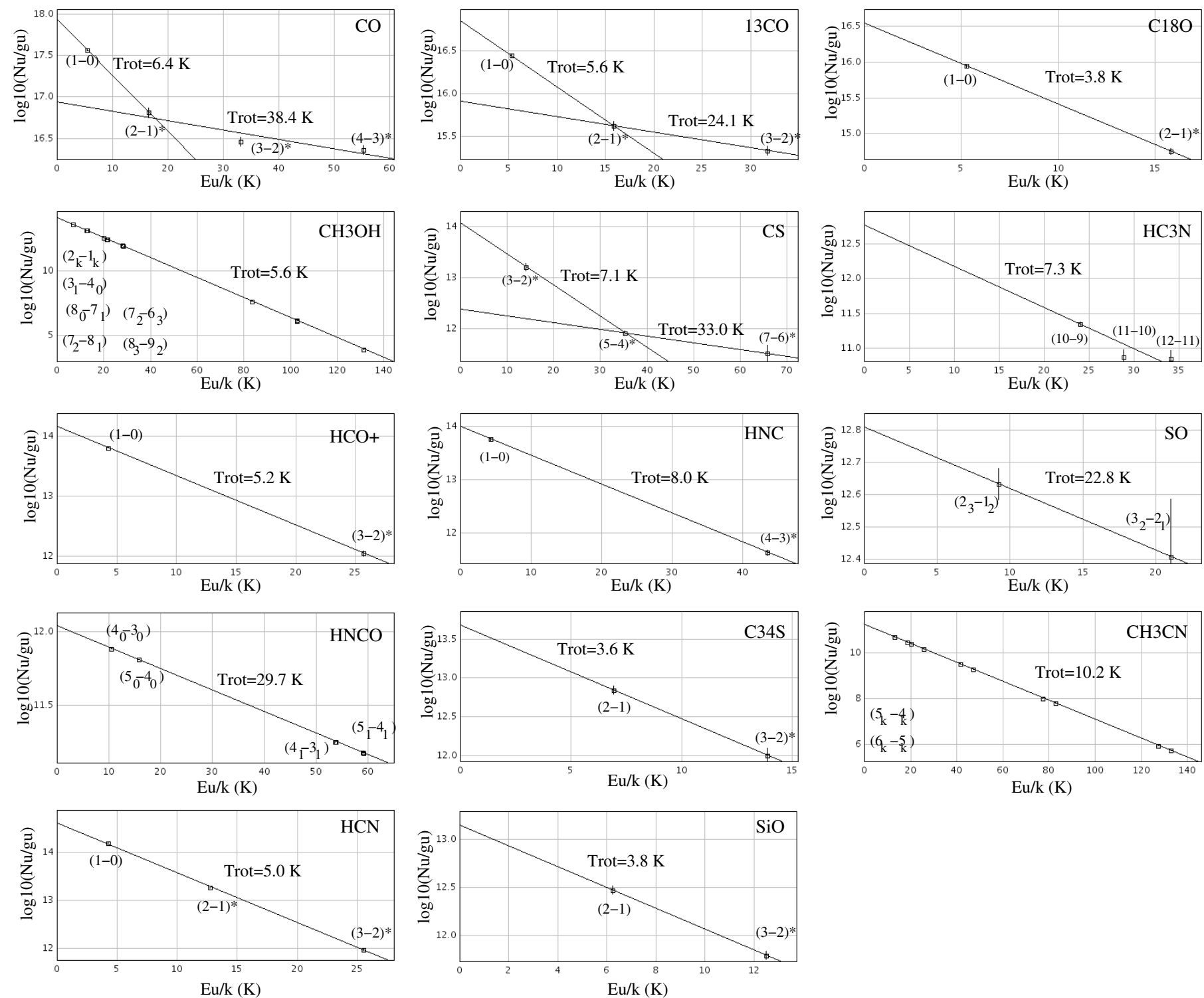

Fig. A.3. Boltzmann diagrams of the molecules observed in the survey for which we have detected more than one transition, or for which we have taken other transitions from the literature (marked with $*$ ). The resulting rotational temperatures are indicated. 


\section{Appendix B: Gaussian fits parameter results}

Table B.1. Parameters fixed during the Gaussian fit have zero errors associated. Synthetic fits do not give errors to the integrated area. Remarks: (b) blended line; $(m)$ multi-transition line; $(h f)$ hyperfine transition; $(s)$ synthetic Gaussian fit using MASSA. For those transitions showing several components, the parameters refer to the main component of the group.

\begin{tabular}{|c|c|c|c|c|c|c|}
\hline Line & $\begin{array}{c}\text { Frequency } \\
\mathrm{MHz}\end{array}$ & $\begin{array}{c}I \\
\mathrm{~K} \mathrm{~km} \mathrm{~s}^{-1}\end{array}$ & $\begin{array}{c}\text { Position } \\
\mathrm{km} \mathrm{s}^{-1}\end{array}$ & $\begin{array}{l}\text { Width } \\
\mathrm{km} \mathrm{s}^{-1}\end{array}$ & $\begin{array}{c}T_{\text {peak }} \\
\mathrm{mK}\end{array}$ & Comments \\
\hline $\mathrm{H}^{13} \mathrm{CN}(1-0)$ & 86340.2 & $0.4 \pm \ldots$ & $1066.3 \pm 0.0$ & $166.4 \pm 0.0$ & 2.4 & hf, s \\
\hline $\operatorname{HCO}\left(1_{1,0}-0_{1,0}\right)$ & 86670.8 & $0.2 \pm \ldots$ & $1100.0 \pm 0.0$ & $240.0 \pm 0.0$ & 0.6 & $\mathrm{~b}, \mathrm{~s}$ \\
\hline $\mathrm{H}^{13} \mathrm{CO}^{+}(1-0)$ & 86754.3 & $0.5 \pm \ldots$ & $1100.0 \pm 0.0$ & $240.0 \pm 0.0$ & 1.9 & $\mathrm{~b}, \mathrm{~s}$ \\
\hline $\mathrm{SiO}(2-1)$ & 86847.0 & $0.6 \pm \ldots$ & $1100.0 \pm 0.0$ & $259.1 \pm 0.0$ & 2.2 & $\mathrm{~b}, \mathrm{~s}$ \\
\hline $\mathrm{HN}^{13} \mathrm{C}(1-0)$ & 87090.8 & $0.2 \pm 0.3$ & $1075.7 \pm 124.4$ & $151.8 \pm 235.9$ & 1.1 & \\
\hline $\mathrm{C}_{2} \mathrm{H}(1-0)$ & 87316.9 & $3.1 \pm \ldots$ & $1098.4 \pm 0.0$ & $240.0 \pm 0.0$ & 12.0 & hf, s \\
\hline $\operatorname{HNCO}\left(4_{0,4}-3_{0,3}\right)$ & 87925.2 & $0.1 \pm \ldots$ & $1100.0 \pm 0.0$ & $230.0 \pm 0.0$ & 0.4 & $\mathrm{~m}, \mathrm{~s}$ \\
\hline $\mathrm{HCN}(1-0)$ & 88631.8 & $11.1 \pm \ldots$ & $1120.1 \pm 0.0$ & $238.5 \pm 0.0$ & 43.0 & hf, s \\
\hline $\mathrm{HCO}^{+}(1-0)$ & 89188.6 & $13.8 \pm 0.3$ & $1122.6 \pm 1.2$ & $230.9 \pm 1.5$ & 55.9 & \\
\hline $\mathrm{HOC}^{+}(1-0)$ & 89487.4 & $0.2 \pm 0.1$ & $1173.3 \pm 17.5$ & $128.2 \pm 51.6$ & 1.8 & \\
\hline $\mathrm{HNC}(1-0)$ & 90663.6 & $7.5 \pm 0.6$ & $1141.3 \pm 0.3$ & $235.9 \pm 3.2$ & 30.8 & \\
\hline $\mathrm{HC}_{3} \mathrm{~N}(10-9)$ & 90979.0 & $1.1 \pm 0.1$ & $1142.6 \pm 10.0$ & $263.7 \pm 24.9$ & 3.8 & \\
\hline $\mathrm{CH}_{3} \mathrm{CN}\left(5_{k}-4_{k}\right)$ & 91987.0 & $0.1 \pm \ldots$ & $1100.0 \pm 0.0$ & $150.0 \pm 0.0$ & 0.6 & $\mathrm{~m}, \mathrm{~s}$ \\
\hline $\mathrm{N}_{2} \mathrm{H}^{+}(1-0)$ & 93173.7 & $1.0 \pm \ldots$ & $1164.1 \pm 0.0$ & $233.5 \pm 0.0$ & 4.0 & $\mathrm{~m}, \mathrm{~s}$ \\
\hline$C^{34} S(2-1)$ & 96412.9 & $0.7 \pm 0.1$ & $1091.2 \pm 15.7$ & $249.0 \pm 29.5$ & 2.7 & \\
\hline $\mathrm{CH}_{3} \mathrm{OH}\left(2_{k}-1_{k}\right)$ & 96741.4 & $0.9 \pm \ldots$ & $1117.5 \pm 0.0$ & $247.2 \pm 0.0$ & 3.3 & $\mathrm{~m}, \mathrm{~s}$ \\
\hline $\mathrm{CS}(2-1)$ & 97981.0 & $\ldots$ & $\ldots$ & $\ldots$ & $\ldots$ & $\mathrm{b}$ \\
\hline $\mathrm{SO}\left(2_{1}-3_{2}\right)$ & 99299.9 & $0.4 \pm 0.1$ & $1111.1 \pm 7.5$ & $136.0 \pm 17.0$ & 3.1 & \\
\hline $\mathrm{SO}\left(5_{4}-4_{4}\right)$ & 100029.6 & $0.2 \pm 0.1$ & $1111.1 \pm 0.0$ & $136.0 \pm 0.0$ & 1.6 & $\mathrm{~b}$ \\
\hline $\mathrm{HC}_{3} \mathrm{~N}(11-10)$ & 100076.4 & $0.5 \pm \ldots$ & $1142.6 \pm 0.0$ & $263.0 \pm 0.0$ & 2.0 & $\mathrm{~b}, \mathrm{~s}$ \\
\hline${ }^{13} \mathrm{CN}\left(1_{1,0}-0_{1,1}\right)$ & 108426.9 & $0.1 \pm \ldots$ & $1104 . \pm 0.0$ & $257.2 \pm 0.0$ & 0.2 & $\mathrm{~m}, \mathrm{~s}$ \\
\hline${ }^{13} \mathrm{CN}\left(1_{2,1}-0_{1,0}\right)$ & 108651.3 & $0.2 \pm \ldots$ & 1104. \pm 0.0 & $257.2 \pm 0.0$ & 0.6 & $\mathrm{~m}, \mathrm{~s}$ \\
\hline $\mathrm{HC}_{3} \mathrm{~N}(12-11)$ & 109173.6 & $0.7 \pm \ldots$ & $1142.6 \pm 0.0$ & $263.0 \pm 0.0$ & 2.6 & $\mathrm{~b}, \mathrm{~s}$ \\
\hline $\mathrm{SO}\left(3_{2}-2_{1}\right)$ & 109252.2 & $0.2 \pm 0.1$ & $1111.1 \pm 0.0$ & $136.0 \pm 0.0$ & 1.3 & $\mathrm{~b}$ \\
\hline $\operatorname{HNCO}\left(5_{1,5}-4_{1,4}\right)$ & 109496.0 & $0.04 \pm \ldots$ & $1100.0 \pm 0.0$ & $230.0 \pm 0.0$ & 0.2 & $\mathrm{~m}, \mathrm{~s}$ \\
\hline $\mathrm{C}^{18} \mathrm{O}(1-0)$ & 109782.2 & $3.6 \pm 0.2$ & $1142.2 \pm 6.1$ & $230.0 \pm 0.0$ & 13.0 & \\
\hline $\operatorname{HNCO}\left(5_{0,5}-4_{0,4}\right)$ & 109905.8 & $0.3 \pm \ldots$ & $1100.0 \pm 0.0$ & $230.0 \pm 0.0$ & 1.3 & $\mathrm{~m}, \mathrm{~s}$ \\
\hline${ }^{13} \mathrm{CO}(1-0)$ & 110201.4 & $12.8 \pm 0.7$ & $1105.2 \pm 3.6$ & $252.1 \pm 4.9$ & 45.8 & \\
\hline $\operatorname{HNCO}\left(5_{1,4}-4_{1,3}\right)$ & 110298.1 & $0.2 \pm \ldots$ & $1100.0 \pm 0.0$ & $230.0 \pm 0.0$ & 0.7 & $\mathrm{~m}, \mathrm{~b}, \mathrm{~s}$ \\
\hline $\mathrm{CH}_{3} \mathrm{CN}\left(6_{k}-5_{k}\right)$ & 110383.5 & $0.1 \pm \ldots$ & $1100.0 \pm 0.0$ & $150.0 \pm 0.0$ & 0.8 & $\mathrm{~m}, \mathrm{~b}, \mathrm{~s}$ \\
\hline $\mathrm{CN}\left(1_{0,2}-0_{0,1}\right)$ & 113191.3 & $4.5 \pm \ldots$ & $1152.8 \pm 0.0$ & $247.4 \pm 0.0$ & 17.3 & $\mathrm{~s}$ \\
\hline $\mathrm{CN}\left(1_{0,2}-0_{0,1}\right)$ & 113491.0 & $12.1 \pm \ldots$ & $1152.8 \pm 0.0$ & $247.4 \pm 0.0$ & 46.0 & $\mathrm{~s}$ \\
\hline $\mathrm{NS}\left(3_{1}-2_{1}\right)$ & 115153.9 & $0.4 \pm \ldots$ & $1100.0 \pm 0.0$ & $140.0 \pm 0.0$ & 2.8 & $\mathrm{~m}, \mathrm{~b}, \mathrm{~s}$ \\
\hline${ }^{12} \mathrm{CO}(1-0)$ & 115271.2 & $172.2 \pm 4.5$ & $1168.3 \pm 0.3$ & $251.5 \pm 0.4$ & 618.0 & b \\
\hline NS $\left(3_{1}-2_{1}\right)$ & 115556.2 & $0.4 \pm \ldots$ & $1100.0 \pm 0.0$ & $140.0 \pm 0.0$ & 2.8 & $\mathrm{~m}, \mathrm{~s}$ \\
\hline
\end{tabular}

